\title{
Floristic List of Tohma (Malatya-Sivas, Turkey) Valley
}

\author{
Tohma Vadisi (Sivas-Malatya, Türkiye) Flora Listesi
}

Research Article

Şükrü Karakuş and Birol Mutlu

Inönü University, Faculty of Arts and Science, Department of Biology, Malatya. Turkey.

\section{A BSTRACT}

$\mathrm{T}$

he list of the vascular plants specimens of Tohma valley (Malatya-Sivas) was given in this study. 1816 plant specimens were collected from the research area between $2007-2015$ years. At the end of the identification of the collected specimens and the results of previous studies, 81 families, 353 genera, 847 species, 18 subspecies, 6 varieties and 871 taxa in total were determined. The largest 3 families are Asteraceae (102 species), Brassicaceae (78 species) and Fabaceae (77 species). The largest 4 genera are Astragalus (31 species), Alyssum (17 species), Onosma (14 species) and Salvia (14 species). The phytogeographical spectrum of the species is as follows: Irano-Turanian elements 298 (35.18\%), Mediterranean elements 39 (4.60\%) and Euro-Siberian elements 27 (3.18\%) and multi-regional or phytogeographical unknown 483 (57.02\%). The number of endemic species in the research area was determined as 169 (20\%). At the same time, new IUCN categories of twelve taxa were proposed.

\section{Key Words}

Darende, Flora, Gürün, IUCN, Tohma.

\section{öz}

u çalışmada Tohma vadisi (Malatya-Sivas) damarlı bitkilerin listesi verilmiştir. 2007-2015 yılları arasında çalışma alanından 1816 bitki örneği toplanmıştır. Toplanan örneklerin ve daha önceki çalışma sonuçlarının değerlendirilmesi sonucunda 81 familya, 353 cins, 847 tür, 18 alttür 6 varyete ve toplamda 871 takson tespit edilmiştir. En büyük üç familya Asteraceae (102 tür), Brassicaceae (78 tür) ve Fabaceae (77 tür)'dir. En büyük 4 cins Astragalus (31 tür), Alyssum (17 tür), Onosma (14 tür) ve Salvia (14 tür) dır. Fitocoğrafik bölgelere göre türlerin dağılımı; Iran-Turan elementi 298 (\%35.18), Akdeniz elementi 39 (\%4.60), Avrupa-Sibirya elementi 27 (\%3.18) ve çok bölgeli ya da fitocoğrafik bölgesi bilinmeyen 483 (\%57.02)'dür. Çalışma alanındaki endemik tür sayısı 169 (\%20) olarak belirlenmiştir. Aynı zamanda 12 takson için yeni IUCN ketegorileri önerilmiştir.

\section{Anahtar Kelimeler}

Darende, Flora, Gürün, IUCN, Tohma.

Article History: Received: May 12, 2016; Revised: Sep 15, 2016; Accepted: Oct 20, 2016; Available Online: Apr 1, 2017.

Dol: 10.15671/HJBC.2017.146

Correspondence to: B. Mutlu, İnönü University, Faculty of Arts and Science, Department of Biology,-Malatya. Turkey. 


\section{INTRODUCTION}

Ctudies on "Turkey's Important Plant Areas" $\checkmark$ have been dated back to the early 1990s. The results of the work of 40 scientists from about 20 universities in Turkey were determined 122 important plant areas [1]. Tohma valley is one of the Important Plant Areas. Therewithal, there are two naturel protected area in the Tohma valley. These areas are Günpınar Waterfall and Somuncubaba Canyon which was declared for naturel protected area with the 19813 numbered of official newspaper in 1988 [2]. For this reason, the area has gained importance in the study of vascular plant biodiversity.

Tohma valley is located between Incesu village (Gürün district of Sivas) and Balaban village (Darende district of Malatya provinces). The area is situated at latitude $38^{\circ}-35^{\circ} \mathrm{N}$ and longitude $37^{\circ}-36^{\circ} \mathrm{E}$. The study area is located in the IranoTuranian phytogeographic region. This area is situated in the B6 square according to Davis' grid system [3]. The altitude of the study area varies from 920-1980 m.

Tohma Valley, starting from Sivas territory into two branches, forms a wide groove to Malatya. The rocks in the valley are formed by the overlapping of the plate-shaped. There are limestone rocks with a height of 300-350 meters on both sides of the valley. The most important feature of the area is of gypsum and the limestone hills.

The climatic characteristics of the area were estimated according to the data from Balaban, Darende, and Gürün meteorological stations. Drought index of Emberger ( $\mathrm{S}=\mathrm{PE} / \mathrm{M}$ ) is 0.94 [4] and Mediterranean climate is seen in the study area.

\section{MATERIALS and METHODS}

The materials of this study are 1816 vascular plant specimens that were collected from Tohma Valley between the 2007 and 2015. At least one sample for each taxon is deposited at INU herbarium.

Specimens were identified basically using the Flora of Turkey [3,5,6], Flora Europaea [7] and description of the new species [8-10]. Identified species, records in the studied area from Flora of Turkey $[3,5,6]$ and other studies $[11,12]$ used in the writing of the floristic list. In the floristic list, the following details are stated: family, genus, species (cultivated plants are indicated an asterisk), authors (abbreviations follow Brummitt and Powell [13]), Turkish name of taxa in quotation marks (according to Güner et al. [14]) locality number, collector(s), collector number, phytogeographical region, endemism and IUCN risk categories [15,16].

Collecting localities in the study area are as follows: (1) Malatya: Darende, Balaban village, 920 m; (2) Malatya: Darende, 3. km from Balaban village to Darende, 930 m; (3) Malatya: Darende, between Yeşiltaş-Balaban village, 950 m; (4) Malatya: Darende, Karaoguz village, 920 m; (5) Malatya: Darende, Yenice village, 960 m; (6) Malatya: Darende, Akçatoprak village 920 m; (7) Malatya: Darende, Akova village, 930 m; (8) Malatya: Darende, Irmaklı village, 920 m; (9) Malatya: Darende, 2. km from Irmaklı village, 950 m; (10) Malatya: Darende, opposite slopes of Irmaklı village, 1400-1800 m; (11) Malatya: Darende, Karşıyaka village 950 m; (12) Malatya: Darende district, 1050 m, 950 m; (13) Malatya: Darende, Günpınar waterfall, 1350 m; (14) Malatya: Darende, between Günpınar waterfallFatmaderviş village, 1300 m; (15) Malatya: Darende, Gedikağzı village 1100 m; (16) Malatya: Darende, 2. km from Darende to Gürün, 1300 m; (17) Malatya: Darende, 8. km from Darende to Gürün, 1300 m; (18) Malatya: Darende, 12. km from Darende to Gürün district, step, 1350 m; (19) Malatya: Darende, Çukurkaya village, 1000-1200 m; (20) Sivas: Gürün, Yazıköy village, 1200 m; (21) Sivas: Gürün, Ağaçlı village, 1200-1400 m; (22) Sivas: Gürün, Bahçeiçi village, 1300 m; (23) Sivas: Gürün, 3. km from Gökpınar lake, 1750 m; (24) Sivas: Gürün, opposite the slopes of Gökpınar lake, 1800 m; (25) Sivas: Gürün, Suçatı village, 1450 m; (26) Sivas: Gürün district, 1400 m; (27) Sivas: Gürün 1. km from Gürün to Sularbaşı village 1500 m; (28) Sivas: Gürün, Tepecik village, 1650 m; (29) Sivas: Gürün, Kavak village, 1800 m; (30) Sivas: Gürün, between Gürün-Incesu village, 1650 m; (31) Sivas: Gürün, Şuğul valley, 1650 m, (32) Sivas: Gürün, Incesu village, Reşadiye town, 1700 m; (33) Sivas: Gürün, 1. km from Yaylacık village, $1750 \mathrm{~m}$. 
The abbreviations used in the text and the floristic list are as follows: Ir.-Tur.: IranoTuranian; Medit.: Mediterranean; E.Medit.: East Mediterranean; Eur.-Sib.: Euro-Siberian; End.: Endemic; CR: Criticaly EN: Endangered; VU: Vulnerable; LR: Lower risk; ŞK: Şükrü Karakuş; INU: İönü University Department of Biology Herbarium in Malatya.

Also in this studies, we have applied the IUCN criteria for the threaten categories of species [14]. Threaten categories of some species are firstly determined.

\section{RESULTS and DISCUSSION}

At the end of identification of 1816 vascular plant specimens, collected in this study and evaluated previous studies were determined in 81 families, 353 genera, 847 species, 18 subspecies, 6 varieties and total 871 taxa. Five species are nonflowering vascular plants. Seven species belong to Gymnospermae, while the other 835 were Angiospermae. Dicotyledones and Monocotyledones are consisted of 721 and 114 species respectively.

The richest families in terms of number of species are Asteraceae (102 species), Brassicaceae (78 species), Fabaceae (77 species), Poaceae (58 species) and Lamiaceae (56 species). The largest genera are Astragalus (31 species), Alyssum (17 species), Onosma (14 species), Salvia (14 species) and Silene (13 species). Largest family and genus indicated that dominant vegetation type of the study area is steppe.

The species of the study area, categorized according to phytogeographical regions, can be listed as follows: Irano-Turanian elements 298 (35.18\%), Mediterranean elements 39 (4.60\%) and Euro-Siberian elements 27 (3.18\%) and multiregional or phytogeographical unknown 483 (57.02\%). The dominance of the Ir.-Tur. elements would be expected because the study area is in the Ir.-Tur. region.

In the study area, 169 taxa are endemic to Turkey and the ratio of endemism is $20 \%$. Distribution to the threatened category of endemic (169) and nonendemic (9) taxa are as follows; 8 taxa CR, 22 taxa EN; 27 taxa VU, 121 taxa LR.
Threaten category of Astragalus darendensis "Darende geveni" have not been determined until this studies. In our observations, we determined that extent of occurrence estimated to be less than $10 \mathrm{~km}^{2}$, severely fragmented or known to exist at only a single location, decline number of mature individuals and population size estimated to number fewer than 50 mature individuals for this species. Acording to IUCN Red List criteria [16], threaten category of the species is proposed as CR B2ab(v);D.

Threaten category of Astragalus malatyaensis "malatya geveni" is $\mathrm{VU}$ in redlist of plant of Turkey [15]. In our observations, we determined that population size estimated to number fewer than 50 mature individuals for this species. Acording to IUCN Red List criteria [16], threaten category of the species is proposed as CR D.

Threaten category of Astragalus scabrifolius "gövdesiz geven" is EN in redlist of plant of Turkey [15]. In our observations, we determined that extent of occurrence estimated to be less than $100 \mathrm{~km}^{2}$, severely fragmented or known to exist at only a single location and decline number of mature individuals for this species. Acording to IUCN Red List criteria [16], threaten category of the species is proposed as CR B1ab(v).

Threaten category of Psephellus brevifimbriatus "has tülübaş" is $C R$ in redlist of plant of Turkey [15]. In our observations, we determined that extent of occurrence estimated to be less than $100 \mathrm{~km}^{2}$, severely fragmented or known to exist at only a single location and decline number of mature individuals for this species. Acording to IUCN Red List criteria [16], threaten category of the species is proposed as CR B1ab(v).

Threaten category of Taraxacum rupicolum "çıtlığ" have not been determined until this studies. In our observations, we determined that extent of occurrence estimated to be less than $10 \mathrm{~km}^{2}$, severely fragmented or known to exist at only a single location and decline number of mature individuals for this species. Acording to IUCN Red List criteria [16], threaten category of the species is proposed as CR B2ab(v). 
Eight endemic species are included in CR (critically) threaten categories which they are Alyssum haussknechtii Boiss. "maraş kevkesi", Astragalus darendensis "Darende geveni", Astragalus malatyaensis "malatya geveni", Astragalus scabrifolius "gövdesiz geven", Psephellus brevifimbriatus "has tülübaş", Taraxacum rupicolum "çıtlığ", Thymus cappadocicus "taş kekiği" and Thymus pallasicus "boz kekik".

Threaten category of Erodium aytacii "bey iğneliği" is suggested as EN by Yıldırımlı and Koca [9]. In our observations, we determined that area of occupancy estimated to be less than $500 \mathrm{~km}^{2}$, severely fragmented or known to exist at no more than five locations and decline of number of mature individuals. Acording to IUCN Red List criteria [16], threaten category of the species is proposed as EN B2ab(v).

Threaten category of Klasea bornmuelleri "yitik topbaş" is DD in redlist of plant of Turkey [15]. In our observations, we determined that Population size estimated to number fewer than 2,500 mature individuals and due to road widening works estimated continuing decline of at least $20 \%$ within five years or two generations for this species. Acording to IUCN Red List criteria [16], threaten category of the species is proposed as EN C1.

Threaten category of Minuartia corymbulosa var. gypsophiloides "kırk tıstıs" is EN in redlist of plant of Turkey [15]. In our observations, we determined that area of occupancy estimated to be less than $500 \mathrm{~km}^{2}$, severely fragmented or known to exist at no more than five locations and decline of number of mature individuals. Acording to IUCN Red List criteria [16], threaten category of the species is proposed as EN B2ab(v).

Threaten category of Salvia ballsiana "gerger şalbası" have not been determined until this studies. In our observations, we determined that area of occupancy estimated to be less than $500 \mathrm{~km}^{2}$. severely fragmented or known to exist at no more than five locations and decline of number of mature individuals. Acording to IUCN Red List criteria [16], threaten category of the species is proposed as EN B2ab(v).

Twenty one endemic species are included in EN (endangered) threaten categories which they are Acanthophyllum oppositiflorum "has takacak", Aethionema lepidioides "tere kayagülü", Arenaria sivasica "sivas kumotu", Astragalus stenosemioides "zirve geveni", Echinophora lamondiana "kaba çördük", Erodium aytacii "bey iğneliği", Erysimum echinellum "siğilli zarife", Fumana trisperma "üç güneşotu", Gypsophila leucochlaena "darende çöveni", Haplophyllum myrtifolium "murt sedosu", Hedysarum pycnostachyum "Fırat batalağı", Hedysarum rotundifolium "özge batalak", Helianthemum germanicopolitanum "özge güngülü", Hesperis bicuspidata "gecemenekşesi", Isatis undulata "etekli çiviotu" Klasea bornmuelleri "yitik topbaş", Minuartia corymbulosa var. gypsophiloides "kırk tıstıs", Onobrychis stenostachya subsp. krausei "başak korunga", Onosma bornmuelleri "Amasya şincarı", Onosma sorgerae var. sorgerae "nemrut çiçeği" and Salvia ballsiana "gerger şalbası".

Threaten category of Acantholimon strigillosum "narin kirpiotu" is VU in redlist of plant of Turkey [15]. In our observations, we determined that area of occupancy estimated to be less than $2,000 \mathrm{~km}^{2}$, severely fragmented or known to exist at no more than 10 locations and decline extent of occurrence for this species. Acording to IUCN Red List criteria [16], threaten category of the species is proposed as VU B2ab(i).

Threaten category of Paronychia cataonica "gürün etyaranı" is VU in redlist of plant of Turkey [15]. In our observations, we determined that area of occupancy estimated to be less than $2,000 \mathrm{~km}^{2}$, severely fragmented or known to exist at no more than 10 locations and decline area of occupancy for this species. Acording to IUCN Red List criteria [16], threaten category of the species is proposed as VU B2ab(ii).

Threaten category of Reseda tomentosa "havlı gerdanlık" is EN in redlist of plant of Turkey [15]. In our observations, we determined that extent of occurrence estimated to be less than $2000 \mathrm{~km}^{2}$, severely fragmented or known to exist at no more than 10 locations, decline of number of mature individuals and population size 
estimated to number fewer than 1,000 mature individuals. Acording to IUCN Red List criteria [16], threaten category of the species is proposed as VU B2ab(v);D1.

Nineteen endemic species are included in VU (vulnarable) threaten categories which they are Acantholimon acerosum var. parvifolium "pişik keveni", Acantholimon strigillosum "narin kirpiotu", Alyssum anatolicum "ana kuduzotu", Alyssum bornmuelleri "seyyah kevkesi", AstragaIus zahlbruckneri "kubbe geveni", Bupleurum papillosum "tüylü şeytanayağı", Cyclotrichium niveum "külotu", Gypsophila pinifolia "hıskıdım", Hedysarum aucheri "altın batalak", Hesperis syriaca "arapıtırı", Minuartia rimarum var. multiflora "yer tıstısı", Onosma argentata "gümüş emcek", Papaver persicum subsp. fulvum "kaşıklık", Phlomis physocalyx "küfe çalbası", Pimpinella flabelliifolia (Boiss.) "yel anasonu", Paronychia cataonica "gürün etyaranı", Reseda tomentosa var. tomentosa "havlı gerdanlık", Tanecatum densum subsp. sivasicum "kırk pireotu" and Tchihatchewia isatidea "allıgelin".

Nine nonendemic taxa were evaluated in threaten category by Ekim et al. [15]. One of them is EN category that it is Scutellaria orientalis subsp. cretacea "kulaklı kaside". Eight of them are VU category that they are Allium asclepideum "koç soğanı", Allium asperiflorum "beyaz soğan", Brassica deflexa "sarkık lahana", Centaurea cassia subsp. cassia "kelseçebaşı", Erodium gaillardotii "bozkır iğneliği", Matthiola ovatifolia "takkeli şebboy", Onosma decorticans "göreme emceği" and Veronica polifolia "dallı maviş".

Floristic list is given below;

1. PTERIDOPHYTA/EĞRELTi BÖLÜMÜ (4 family/4 genus/5 species)

\section{ASPLENIACEAE/SAÇAKOTUGILLER (1 genus} /1 species)

Asplenium haussknechtii Godet \& Reut. "karabacak", (31), 23.04.2009, ŞK 978.

\section{EQUISETACEAE/ATKUYRUĞUGILLER (1/2)}

Equisetum arvense L. "atkuyruğu", (7), 16.04.2008, ŞK 165. E. giganteum L. "kırk kilito- tu", (17), 13.06.2008, ŞK 376.

OPHIOGLOSSACEAE/DILOTUGILLER (1/1)

Ophioglossum Iusitanicum L. "dil otu", (31), 23.04.2009, ŞK 927.

\section{PTERIDACEAE/BALDIRIKARAGILLER (1/1)}

Adiantum capillus-veneris L. "baldırıkara", (13), 06.07.2008, ŞK 655.

\section{PINOPHYTA/AÇIK TOHUMLULAR (3/5/7)}

\section{CUPRESSACEAE/SERVIGILLER (1/2)}

Juniperus excelca M.Bieb. var. excelsa "boz ardıç", (22), 16.04.2008, ŞK 190. J. oxycedrus L. subsp. oxycedrus "katran ardıcı", (24), 21.09.2008, ŞK 820.

\section{EPHEDRACEAE/DENIZÜZÜMÜGILLER (1/1)}

Ephedra major Host. subsp. major "hum", (13), 18.05.2008, ŞK 297.

\section{PINACEAE/ÇAMGILLER (3/4)}

*Cedrus libani A.Rich var. libani "katranağacı", (29), 21.09.2008, ŞK 813. *Picea orientalis (L.) Link."lâdin", (24), 21.09.2008, ŞK 823. *Pinus nigra L. subsp. pallasiana (Lamb.) Holmboe var. pallasiana "karaçam", (12), 13.06.2008, ŞK 377. *P. sylvestris L. var. hamata Steven "sarıçam", (12), 13.06.2008, ŞK 380.

\section{MAGNOLIOPHYTA/KAPALI TOHUMLULAR (74/344/835)}

\section{MAGNOLIOPSIDA/MANOLYA SINIFI $(60 / 289 / 721)$}

\section{ACANTHACEAE/AYIPENÇESIGILLER (1/1)} Acanthus hirsutus Boiss. subsp. hirsutus "kıllı ayıpençesi", (23), 21.06.2009, ŞK 1602, (End., LR).

AMARANTHACEAE/HOROZiBiĞiGiLLER (4/4) Amaranthus albus L. "kömüş mancarı", (15), 02.11.2008, ŞK 835. Chenopodium chenopodioides (L.) Aellen "kaz sirkeni", (17), 21.09.2008, ŞK 798. Halimione verrucifera (M.Bieb.) Aellen "betme" (21), 29.11.2008, ŞK 863. Noaea mucronata (Forssk.) Asch. \& Schweinf subsp. mucronata "hölmezotu", (21), 29.11.2008, ŞK 859. 


\section{APIACEAE/MAYDANOZGILLER (19/35)}

Astrodaucus orientalis (L.) Drude, "havyıldız", (29), B.Post 845, (Ir.-Tur.). Blacunaria microcarpa (M.Bieb.) Pimenov \& V.N.Tikhom "sarunotu", (13), 18.05.2008, ŞK 338. Bupleurum cappadocicum Boiss. "peri şeytanı", (10), 10.05.2014, ŞK 4688. B. croceum Fenzl "çiğdem şeytanı", (19), 13.06.2008, ŞK 455, (Ir.-Tur.). B. eginense (Wolff) Snogerup "eğin şeytanayağı", (10), 24.05.2014, ŞK 4818, (End., LR). B. gerardii All. "çalı şeytanı", (10), 24.05.2014, ŞK 4828. B. heldreichii Boiss. \& Balansa "şeytan tuzu", (32), 06.07.2008, ŞK 544, (Ir.-Tur.), (End., LR). B. papillosum DC. "tüylü şeytanayağı", (19), 13.06.2008, ŞK 451, (Ir.-Tur.), (End., VU). B. sulphureum Boiss. \& Balansa "ters şeytanayağı", (18), 13.06.2008, ŞK 460, (Ir.-Tur.), (End., LR). Daucus carota L. "yabani havuç", (7), 19.07.2008, ŞK 722. Echinophora lamondiana Yıldız \& Bahç. "kaba çördük", (16), B.Yıldız 14000 \& Z.Bahçecioğlu, (Ir.-Tur.), (End., EN). E. tenuifolia L. var. sibthorpiana (Guss.) Tutin "sarıçördük", (21), 29.11.2008, ŞK 858. Falcaria falcarioides (Bornm. \& H.Wolff) H.Wolff "has orakotu", (19), 13.06.2008, ŞK 438. Ferula communis L. subsp. communis "atkasnağı", (7), 19.07.2008, ŞK 728. F. rigidula Fisch. ex DC. "siyabu", (31), 21.06.2009, ŞK 1634, (Ir.-Tur.). Ferulago pauciradiata Boiss. \& Heldr. "etekli kişniş", (17), Hub.-Mor. 12026, (Ir.-Tur.), (End., LR). F. platycarpa Boiss. \& Balansa "çelebi kişnişi", (31), 21.06.2009, ŞK 1637, (Ir.-Tur.), (End., LR). Grammosciadium macrodon Boiss. "koca kami", (12), 07.05.2013 ŞK 3824. Laserpitium hispidum M.Bieb. "saçlı enguban", (19), 13.06.2008, ŞK 435, (Euro.-Sib.). Malabaila secacul (Mill.) Boiss.) subsp. secacul "davarotu", (25), 13.06.2008, ŞK 476. Pimpinella cappadocica Boiss. \& Balansa var. cappadocica "peri anasonu", (21), 19.07.2008, ŞK 763, (Ir.-Tur.), (End., LR). P. flabelliifolia (Boiss.) Benth. \& Hook. ex Drude "yel anasonu", (25), 06.06.2009, ŞK 1407, (Ir.-Tur.), (End., VU). P. rhodantha Boiss. "gül anason", (25), 13.06.2008, ŞK 485. Prangos meliocarpoides Boiss. var. meliocarpoides "sultanteresi", (2), 10.06.2015, ŞK 6088, (Ir.-Tur.), (End., LR). P. pabularia Lindl. "beyik", (13), 06.07.2008, ŞK 638, (Ir.-Tur.). P. peucedanifolia Fenzl "kaya çakşırı", (25), 19.05.2009, ŞK 1149, (Ir-Tur.). Scandix iberica M.Bieb. "atkişneşotu", (19), 13.06.2008, ŞK 454. S. pecten-veneris L. "zühretarağı", (13), 18.05.2008, ŞK 306. S. stel- lata Banks. \& Sol. "dağ kişkişi", (17), 12.05.2008 ŞK 31. Seseli libanotis Koch "dağhavucu", (2), 18.05.2008, ŞK 239. S. tortuosum L. "horozgözü", (31), 06.07.2008, ŞK 562. Simyrnium cordifolium Boiss. "kokarbaldıran", (10), 24.04.2013 ŞK 3668, (Ir-Tur.). Torilis leptophylla (L.) Reich. f. "ince dercikotu", (13), 13.06.2008, ŞK 348.

Turgenia latifolia (L.) Hoffm. "karaheci", (16), 13.06.2008, ŞK 429. Zosima absinthifolia (Vent.) Link "peynirotu", (9), 10.06.2015, ŞK 6093.

\section{APOCYNACEAE/ZAKKUMGILLER" (1/2)}

Vincetoxicum canescens (Willd.) Decne. subsp. canescens "zilasur", (16), 13.06.2008, ŞK 378. V. fuscatum Rchb.f. subsp. boissieri (Kusnezov) Burowicz "gâvur üzerliği", (18), 21.06.2009, ŞK 1480.

\section{ARISTOLOCHIACEAE/LOHUSAOTUGILLER $(1 / 1)$}

Aristolochia maurorum L. "kargabardağı", (14), 13.05.2009, ŞK 1096.

\section{ASTERACEAE/PAPATYAGILLER (47/102)}

Achillea arabica Kotschy. "hanzabel", (16), 13.06.2008, ŞK 409. A. cappadocica Hausskn. \& Bornm. "gırtkesen", (1), 13.06.2009, ŞK 515, (Ir.Tur.), (End., LR). A. cucullata Bornm. "tavukkıçı", (24), 21.06.2009, ŞK 1590, (Ir.-Tur.), (End., LR). A. phrygia Boiss. \& Balansa "özge civanperçemi", (18), D. 21865, (Ir.-Tur.), (End., LR). A. pseudoaleppica Hausskn. ex Hub.-Mor. "harput perçemi", (4), 06.06.2009, ŞK 1383, (Ir.-Tur.), (End., LR). A. santolinoides Lag. subssp. wilhelmsii (K.Koch.) Greuter "kardaşkınası", (9), 18.05.2008, ŞK 17, (Ir.Tur.). Anthemis aciphylla Boiss. var. aciphylla "Iğne papatyası", (17), 12.05.2008, ŞK 58, (E. Medit.), (End., LR). A. anthemiformis (Freyn \& Sint.) Grierson "yayla papatyası", (7), 13.05.2009, ŞK 577, (End., LR). A. cretica L. subsp. subsp. anatolica (Boiss.) Grierson "horoz papatyası", (31), 13.04.2009, ŞK 928. A. fumariifolia Boiss. "çorak papatyası", (13), 06.07.2008, ŞK 577, (Ir.-Tur.), (End., LR). A. kotschyana Boiss. var. discoidea Grierson "koç papatyası", (21), 29.11.2008, ŞK 862. A. pauciloba Boiss. var. pauciloba "bol papatya", (22), 06.07.2008, ŞK 603. Arctium minus (Hill) Bernh. "löşlek", (7), 10.08.2008, ŞK 783, (Euro.Sib.). *Bidens tripartita L., "üç suketeni" (22), 21.09.2008, ŞK 808. Carduus nutans L subsp. leiophyllus (Petrovi) Stoj. \& Stef. "kerbeş", (12), 
18.05.2008, ŞK 234. C. pycnocephalus L. subsp. albidus (M.Bieb.) Kazmi. "eşek soymacı", (12), 18.05.2008, ŞK 204. Carlina oligocephala Boiss. \& Kotschy subsp. oligocephala "domuz dikeni", (4), 21.06.2009, ŞK 1523. Centaurea aegialophila Boiss. \& Heldr. ex Boiss. "kum deligözü", (24), 21.06.2009, ŞK 1596, (E. Medit.). C. armena Boiss. "yer sarıbaşı", (31), 06.07.2008, ŞK 632, (Ir.-Tur.). C. carduiformis DC. subsp. carduiformis var. carduiformis "kavgalaz", (2), 18.05.2008, ŞK 237. C. carduiformis DC. subsp. carduiformis var. thrinciifolia (DC.) Wagenitz "kavgalaz", (1), 19.07.2008, ŞK 697. C. cassia Boiss subsp. cassia "kelseçebaŞı", (19) 19.07.2008, ŞK 442, (E. Medit.) (VU). C. drabifolia Sibth. \& Sm. subsp. floccosa (Boiss.) Wagenitz \& Grauter "çakıl sarıbaş", (21), 13.06.2008, ŞK 501. C. glastifolia L. "kotankıran", (32), 06.07.2008, ŞK 565, (Ir.-Tur.). C. iberica Trev.ex Sprengeln "deligözdikeni", (10), 07.05.2013 ŞK 3901. C. patula DC. "salkım düğme", (31), 06.07.2008, ŞK 624, (Ir.-Tur.). C. pulchella Ledeb. "gümüşsüpürge", (17), 12.05.2007, ŞK 33. C. solstitialis L. subsp. solstitialis "çakır dikeni", (1), 19.07.2008, ŞK 700. C. tomentella Hand.-Mazz. "sivri kavgalaz", (1), 18.05.2008, ŞK 327, (Ir.-Tur.), (End., LR). C. urvillei DC. subsp. hayekiana Wagenitz "yozkötürüm", (21), 13.06.2008, ŞK 486. C. urvillei DC. subsp. nimrodis (Boiss. \& Hausskn) Wagenitz "koçkötürüm", (31), 13.05.2009, ŞK 1147, (Ir.-Tur.). Chardinia orientalis (L.) Kuntze "çağla otu", (27), 06.06.2009, ŞK 1445, (Ir.-Tur.). Chondrilla juncea L. "karakavuk", (13), 06.07.2008, ŞK 649. Cichorium intybus L. "hindiba", 19.07.2008, ŞK 721. Cirsium arvense (L.) Scop. "köygöçüren", (22), 06.07.2008, ŞK 609. C. leucocephalum (Willd.) Spreng. subsp. leucocephalum "hamurkesem", (2), 29.11.2008, ŞK 856. C. libanoticum DC. subsp. arachnoideum P.H.Davis \& Paris "öküz kazankulpu", (24), 21.09.2008, ŞK 829, (Ir.-Tur.). Cnicus benedictus L. "topdiken", (25), 13.04.2009, ŞK 954. Cota austriaca (Jacq.) Sch.Bip. "Bavuçça", (10), 10.05.2014, ŞK 4680. C. tinctoria (L.) J.Gay ex Guss. var. tinctoria "boyacı papatyası", (13), 18.05.2008, ŞK 322. C. wiedemanniana (Fisch. \& C.A.Mey) Holub, "babuçça", (12), Sorger 71-42-2. Cousinia cataonica Boiss. \& Hausskn. "kırkkızan", (21), 13.06.2008, ŞK 487, (Ir.-Tur.), (End., LR). Crepis foetida L. subsp. rhoeadifolia (M.Bieb.) elak. "sakarkanak", (16), 21.09.2008, ŞK 796. C. sancta (L.) Bornm. subsp. nemausensis
(P.Fourn.) Babc. "yaban kıskısı", (17), 12.05.2008, ŞK 29. Crupina crupinastrum (Moris) Vis. "gelindöndüren", (16), 13.06.2008 ŞK 363. C. vulgaris Cass. "kır gelindöndüreni", (10), 07.05.2013 ŞK 3908. Cyanus depressus (M.Bieb.) Soják "gökbaş", (12), 18.05.2008, ŞK 201. C. pichleri (Boiss.) Holub subsp. pichleri "düğmeli ot", (18), 03.05.2009, ŞK 1009. Echinops adenocaulos Boiss. "şeytan değirmeni", (13), 06.07.2008, C.Vural 4379, (Ir.Tur.). E. chardinii Boiss. "kelle dikeni", (20), 05.08.2007, C.Vural 4380, (Ir.-Tur.). E. pungens Trautv. var. pungens "bongıl", (28), 13.06.2008 ŞK 507, (Ir.-Tur.). E. spinosissimus Turra subsp. spinosissimus "eşekköftesi", (1), 19.07.2008, ŞK 702. Erigeron acris L. subsp. acris "mavi şifaotu", (13), 06.07.2008, ŞK, 674. Flago arvensis L. "keçeotu", 45, 06.06.2009, ŞK 1426. Gundelia tournefortii L. var. tournefortii "kenger", (10), 18.05.2006, ŞK 273. *Helianthus annuus L. "ayçiçeği", (17), 21.09.2008, ŞK 791. Helichrysum arenarium (L.) Moench subsp. aucheri (Boiss.) P.H.Davis \& Kupicha "yayla gülü", (24), 21.06.2009, ŞK 1588, (Ir.-Tur.), (End., LR). H. arenarium (L.) Moench subsp. rubicundum (C.Koch) P.H.Davis \& Kupicha "yayla"gülü", (25), 13.06.2008, ŞK 483. H. chionophilum Boiss. \& Balansa, "yayla hencecaliği", (24), 06.06.2009, ŞK 1588, (End., LR). $\boldsymbol{H}$. noeanum Boiss. "gülüzar", (9), 21.06.2009, ŞK 1516, (Ir.-Tur.), (End., LR). H. pallasi (Spreng.) Ledeb. "kocamançiçeği", (13), 06.12.2008, ŞK 875, (Ir.-Tur). H. plicatum DC. subsp. plicatum "mantuvar", (13), 06.07.2008, ŞK 642. Hypochoeris radicata L. "dağmarulu", (33), 13.05.2009, ŞK 1068, (Euro.-Sib.). Inula germanica L. "ekin andızotu", (7), 19.07.2008, ŞK 725, (Euro.-Sib.). Jurinea consanguinea DC. "geyikgöbeği", (13), 18.05.2008, ŞK 318. Klasea bornmuelleri (Azn.) Greuter \& Wagenitz "yitik topbaş", (9), 03.05.2009, ŞK 1011, (Ir.Tur.), (End., EN A4a;C1). K. oligocephala (DC.) Greuter \& Wagenitz "saplı topbaş", (10), 24.05.2014, ŞK 5052, (Ir.-Tur.), (End., LR). Lactuca serriola L. "eşekhelvası", (7), 19.07.2008, ŞK 724, (Euro.-Sib.). L. tuberosa Jacq. "topar marul", (16), 3.06.2008, ŞK 364. Leontodon asperrimus (Willd.) Endl. "aşyemliği", (16), 13.06.2008, ŞK 374, (Ir.-Tur.). L. crispus Vill. subsp. asper (Waldst. \& Kit.) Röhl. var. asper "aslandişi", (16), 13.06.2008, ŞK 418. L. oxylepis Boiss. \& Heldr. "bayır aslandişi", (21), 13.06.2008, ŞK 495, (Ir.-Tur.), (End., LR). Onopordum polycephalum Boiss. "beyaz kangal", (12), 
Sorger 7148, (Ir.-Tur.), (End., LR). Picnomon acarna (L.) Cass. "kılçıkdiken", (7), 19.07.2008, ŞK 716. Picris kotschyi Boiss. "arap şirosu", (19), 13.06.2008, ŞK 457. Pilosella cymosa (L.) F.W.Schultz \& Sch.Bip. "sülün tırnakotu", (13), 06.07.2008, ŞK 644. Psephellus brevifimbriatus (Hub.-Mor.) Wagenitz. "has tülübaş", (10), 28.05.2009, ŞK 1195, (Ir.-Tur.), (End., CR B1ab(v)). Ptilostemon afer (Jacq.) Greuter subsp. eburneus Greuter "has bozlanotu", (23), 06.07.2008, ŞK 566, (End., LR). Pulicaria dysenterica (L.) Bernh. subsp. dysenterica "yaraotu", (1), 19.07.2008, ŞK 776. Rhaponticum repens (L.) Hidalgo "kekredikeni", (1), 06.07.2008, ŞK 696, (Ir.-Tur.). Scorzonera cana (C.A.Mey.) Grieb. var. cana "tekesakalı", (10), 24.04.2013 ŞK 3706. S. hieraciifolia Hayek "cıbıl tekesakalı", (7), 13.05.2009, ŞK 1033, (Ir.Tur.), (End., LR). S. incisa DC. "kanık", (11), 12.05.2007, ŞK 92, (Ir.-Tur.). S. pseudolanata Grossh. "keçimemesi", (31), 13.04.2009, ŞK 937, (Ir.-Tur.). S. suberosa K.Koch subsp. suberosa "çalık", (17), 12.05.2008, ŞK 42, (Ir.-Tur.). S. tomentosa L. "alabent", (32), 06.07.2008, ŞK 539, (Ir.-Tur.), (End., LR). Senecio cilicius Boiss. "ak kanaryaotu", (7), 19.07.2008, ŞK 719, (Ir.-Tur.), (End., LR). S. mollis Willd. "su kanaryaotu", (31), 06.07.2008, ŞK 626, (Ir.-Tur.). S. vernalis Waldst \& Kit. "kanaryaotu", (19), 13.06.2008, ŞK 456. Solidago virgaurea L. subsp. virgaurea "altınbaşak çiçeği", (31), 21.06.2009, ŞK 162, (Euro.-Sib.). Sonchus asper (L.) Hill subsp. asper "eşekgevreği", (7), 19.07.2008, ŞK 717. Tanecatum densum (Lab.) Schultz Bip. subsp. sivasicum "kırk pireotu", (27), ŞK 1451, (Ir.-Tur.), (End., VU). Taraxacum microcephaloides Soest "ebemçıtlığı", (13), 06.12.2008, ŞK 873, (Ir.-Tur.), (End., LR). T. revertens G.E.Haglund "caşir", (24), 21.09.2008, ŞK 821, (Ir.-Tur.), (End., LR). T. rupicolum Yıldırım, "çıtığ", (12), 10.09.2009, H.Yıldırım 2207, (End. DD). T. scaturiginosum G.E.Haglund "kıvırkıvır", (8), 18.05.2008 ŞK 268. Tragopogon coloratus C.A.Mey. "katır yemliği", (19), 13.06.2008, ŞK 450, (Ir.-Tur.). T. dubium Scop. "at yemliği", (8), 18.05.2008, ŞK 251. T. porrifolius L. subsp. longirostris (Sch.Bip.) Greuter "helevan", (8), 18.05.2008, ŞK 267. T. pterocarpus DC. "boz yemlik", (31), 19.05.2009, ŞK 1142, (Ir.-Tur.). Tripleurospermum caucasicum (Willd.) Hayek "akbabaotu", (10), 24.04.2013 ŞK 3658. T. decipiens (Fisch. \& C.AMey.) Bornm. "sarı papatya", (8),
16.04.2008, ŞK 145. T. sevanense (Manden.) Pobed. "hanım gödesi", (10), 24.04.2013 ŞK 3660. Xanthium strumarium L. subsp. strumarium "koca pıtrak", (7), 10.08.2008, ŞK 788. Xeranthemum anuum L. "kağıtçiçeği", (13), 06.07.2008, ŞK 685.

\section{BERBERIDACEAE/KARAMUKGILLER (1/1)}

Berberis crataegina DC. "karamuk", (9), 03.05.2009, ŞK 1022.

\section{BORAGINACEAE/HODANGILLER (21/49)}

Alkanna orientalis (L.) Boiss. var. orientalis "sarı sormuk", (12), 03.05.2013 ŞK 3840, (Ir.Tur.). Anchusa azurea Mill. var. azurea "sığırdili", (30), 06.07.2008, ŞK 550. A. strigosa Banks \& Sol. "gelezan", (7), 03.05.2009, ŞK 990. Arnebia decumbens (Vent.) Coss. \& Kralik "eğnik", (10), 24.05.2014, ŞK 4836, (Ir.-Tur.). Asperugo procumbens L. "nevazilotu", (11), 13.04.2009, ŞK 902, (Euro.-Sib.). Buglossoides arvensis (L.) I.M.Johnst. subsp. sibthorpiana (Griseb.) R.Fern. "tarla taşkeseni", (10), 24.04.2013 ŞK 3658. B. incrassata (Guss.) I.M.Johnst. "tok taşkesen", (10), 24.04.2013 ŞK 3710, (Medit). B. tenuiflora (L.f.) I.M.Johnst. “ince taşkesen”, (2), 13.05.2008, ŞK 1052. Cerinthe minor L. subsp. auriculata (Ten.) Domac "livarotu", (13), 18.05.2008, ŞK 312. Cynoglosum montanum L. "dağ köpekdili", (10), 28.05.2008, ŞK 1252. Echium glomeratum Poir. "gül sığırdili", (16), 03.06.2008, ŞK 375, (E. Medit.). E. italicum L. "kurtkuyruğu", (1), 19.07.2008, ŞK 698. (E. Medit.). Heliotropium bovei Boiss. "gelinfesi", (19), 21.09.2008, ŞK 800, (E. Medit.). $\boldsymbol{H}$. circinatum Griseb. "deli bambulotu", (26), McNeill 433, (Ir.-Tur.). H. dolosum De Not. "bambulotu", (13), 06.07.2008, ŞK 680. Heterocaryum rigidum A.DC. "diri gürke", (25) 19.05.2009, ŞK 1151, (Ir.-Tur.). Huynhia pulchra (Roen. \& Schult.) Greuter \& Burdet "güzel eğnik", (13), 16.04.2008, ŞK 130, (Euro.-Sib.). Lappula barbata (M.Bieb.) Gürke "gürke", (24), 22.06.2009, ŞK 1579, (Ir.-Tur.). L. patula (Lehm). Asch. ex Gürke "boz gürke", (25), 23.04.2009, ŞK 956. L. squarrosa (Retz.) Dumort "sülün gürke", (25), 13.06.2008, ŞK 474. Lithospermum arvense L. "taşkesen", (28), 13.06.2008, ŞK 469, (Euro.-Sib.). Macrotomia densiflora (Ledeb.) McBride "koca eğnik", (21), 19.07.2008, ŞK 764, (Ir.-Tur.). Moltkia coerulea (Willd.) Lehm. "mavi kesen", (21), ŞK 496, (Ir.-Tur.). Myosotis 
lithospermifolia Hornem, "taş boncukotu", (2), 12.05.2007. ŞK 48. M. minutiflora Boiss. \& Reut. "minik boncukotu", (23), Sorger 71-49-13, (Medit.). Neatostema apulum I.M.Johnst. "sarıtaşkesen", (13), 13.05.2008, ŞK 1037, (Medit.). Nonea anchusoides Boiss \& Bushe "boz sormuk", (12), 18.05.2008, ŞK 202, (Ir.-Tur.). N. caspica G.Don "bahar sormuğu", (2), 12.05.2007. ŞK 46. (Ir.-Tur.) N. melanocarpa Boiss. "gelin sormuğu", (23), 28.05.2009, ŞK 132, (Ir.-Tur.). N. stenosolen Boiss \& Balansa "sormuk otu", (10), 24.04.2013 ŞK 3717, (Ir.-Tur.), (End., LR). Onosma albo-rosea Fisch \& C.A.Mey subsp. alborosea var. alborosea "kaya emceği", (18), 03.05.2009, ŞK 1003. O. argentata Hub.-Mor. "gümüş emcek", (31), 19.05.2009, ŞK 1135, (Ir.-Tur.), (End., VU). O. auriculata DC. "altın damlası" (29), B.Post 849, (Ir.-Tur.). O. bornmuelleri Hausskn. \& Bornm. "Amasya şincarı", (10), 18.05.2008, ŞK 271, (Ir.-Tur.), (End., EN). O. briquettii Czeczott. "yıldız emziği", (10), 28.05.2009, ŞK 1233, (Ir.-Tur.), (End., LR). O. caeurelescens Boiss. "şincar", (19), 13.05.2009, ŞK 1083, (Ir. Tur.). O. decorticans Riedl. "göreme emceği", (31), 21.06.2009, ŞK 1605, (VU). O. Iycaonica Hub. Mor. "Konya emceği", (12), 03.05.2013 ŞK 3838, (Ir.-Tur.), (End., LR). O. mollis DC. "divanköşk", (19), 13.05.2009, ŞK 1083, (Ir.-Tur.). O. roussaei DC. "yamaç emceği", (27), 06.06.2009, ŞK 1446, (Ir.-Tur.). O. sericeum Willd. "kâğıt emcek", (16), 13.06.2008, ŞK 357, (Ir.-Tur.). O. sorgerae Teppner var. sorgerae "nemrut çiçeği" (24), 26.06.1971, Sorger 71-50-11, (Ir.-Tur.), (End., EN). O. sorgerae Teppner var. subglabriflorum Teppner "nemrut çiçeği", (12), 03.05.2013 Ş.K. 3845, (Ir.-Tur.), (End. LR). O. stenoloba Hausskn. ex Riedl "Tosya emceği", (12), 03.05.2013 ŞK 3844, (Ir.-Tur.), (End., LR). O. tauricum Willd var. taurica "emzikotu", (10), 28.05.2009, ŞK 1133. Paracaryum cappadocicum Boiss \& Bal. "peri çarşağı", (26), 23.04.2009, ŞK 919, (Ir.-Tur.), (End., LR). P. cristatum (Schreb.) Boiss. subsp. cristatum "ana çarşakotu", (13), 18.05.2008, ŞK 337. (Ir.-Tur.), (End., LR). $P$. strictum (K.Koch) Boiss. "meşe çarşakotu", (8), 18.05.2008, ŞK 261, (Ir.-Tur.). Rinderia caespitosa (A.DC.) Bunge "hoş gelin", (10), 03.05.2009, ŞK 1021, (Ir.-Tur.), (End., LR).

\section{BRASSICACEAE/TURPGILLER (34/78)}

Aethionema arabicum (L.) Andrz. ex DC. "arap taşçantası", (31), 23.04.2009, ŞK 972. A. arme- num Boiss. "taş çantası", (25), 13.06.2008, ŞK 482, (Ir.-Tur.). A. capitatum Boiss. \& Balansa "başmürselotu", (10), 24.04.2013 ŞK 3718. A. carneum (Banks \& Sol.) B.Fedtsch. "al kayagülü", (11), 13.04.2009, ŞK 899, (Ir.-Tur.). A. grandiflorum Boiss. \& Hohen. var. grandiflorum "koca kayagülü", (12), 07.05.2013 ŞK 3869. A. iberideum (Boiss.) Boiss. "ak kayagülü", (25), 28.05.2009, ŞK 1385. A. Iepidioides Hub.-Mor. "tere kayagülü", (26), D. 21886, (Ir.-Tur.), (End., EN). A. membranaceum (Desv.) DC. "etekli kayagülü", (18), 13.05.2009, ŞK 1365. A. oppositifolium (Pers.) Hedge "bodur kayagülü", (1), 16.04.2008, ŞK 124. Alyssum anatolicum Hausskn. ex Nyár. "ana kuduzotu", (29), Post 1906: 292, (Ir.-Tur.), (End., VU). A. aureum (Fenzl) Boiss. "altın kuduzotu", (18), 12.05.2007, ŞK 39. A. bornmuelleri Hausskn. ex Degen "seyyah kevkesi", (10), 24.04.2013 ŞK 3713, (Ir.-Tur.), (End., VU). A. condensatum Boiss. \& Hausskn. subsp. condensatum "kuduzotu", (23), 28.05.2009, ŞK 1333. A. dasycarpum Stephan ex Willd. "boz kuduzotu", (11), 30.03.2008 ŞK 98. A. desertorum Stapf. "dumanotu", (11), 30.03.2008, ŞK 97. A. filiforme Nyár. "telli kevke", (21), 13.06.2008, ŞK 491, (Ir.Tur.), (End., LR). A. haussknechtii Boiss. "Maraş kevkesi", (29), Post 1906:292, (End., CR). A. Iepidoto-stellatum (Hausskn \& Bornm.) T.R.Dudley "yıldız kevkesi", (17), D 21861, (Ir.-Tur.), (End., LR). A. linifolium Stephan ex Willd. var. teheranicum Bornm. "çıplak kuzuotu", (1), 16.04.2008, ŞK 115. A. simplex Rudolph "sade kuduzotu" (19), 13.05.2009, ŞK 1028. A. praecox Boiss. \& Balansa "güzel kuduzotu", (19), 13.05.2009, ŞK 1077, (End., LR). A. stapfii Vierh. "acem kuduzotu", (10), 24.04.2013 ŞK 3654, (Ir.-Tur.). A. strigosum Banks \& Sol. subsp. strigosum "dökük kuduzotu", (10), 10.05.2014, ŞK 4697. A. sulphureum T.R.Dudley \& Hub.-Mor. "acı kuduzotu", (16), 13.06.2008, ŞK 430, (Ir.-Tur.). A. szovitsianum Fisch. \& C.A.Mey. "çar kuduzotu", (17), 12.05.2007, ŞK 47. A. xanthocarpum Boiss. "dalsız kevke", (25), 28.05.2009, ŞK 1319. Arabis alpina L. subsp. alpina "kazteresi", (24), 03.05.2009, ŞK 993. A. aucheri Boiss. "cılız tere", (10), 07.05.2013 ŞK 3918. A. nova Vill. "tıfıl kazteresi", (30), 13.04.2009, ŞK 968. Aubrieta canescens (Boiss.) Bornm. var. canescens "obrizya", (18), 03.05.2009, ŞK 1008, (End., LR). Barbarea brachycarpa Boiss. subsp. minor (K.Koch) Parolly \& Eren var. minor "nicarcık", (10), 24.04.2013 ŞK 3728, (Ir.-Tur.). Boreava orientalis Jaub. \& Spach 
"sarıot", (9), 16.04.2008, ŞK 174. Brassica deflexa Boiss. "sarkık lahana", (10), 28.05.2009, ŞK 1209, (Ir.-Tur.) (VU). B. elongata Ehrh. "uzun şalgam", (32), 06.07.2008, ŞK 529. Camelina hispida Boiss. var. hispida "kıllı ketentere", (31), 13.04.2009, ŞK 942. C. microcarpa Andrz. "tarla ketenteresi", (25), 28.05.2009, ŞK 1311. C. rumelica Velen. "ketentere", (17), 12.05.2007, ŞK 63. Capsella bursa-pastoris (L.) Medik. "çobançantası", (1), 30.03.2008, ŞK 88. Chorispora purpurascens (Boiss. \& Sol.) Eig "ala külünk", (1), 30.03.2008, ŞK 94. C. tenella (Pall.) DC. "kokar külünk", (22), 16.04.2008, ŞK 153. Clypeola jonthlapsi L. "akçeo$\mathrm{tu}^{\prime \prime},(17), 12.05 .2007$, ŞK 49. Conringia orientalis (L.) Dumort. "kocatelkari", (17), 12.05.2007, ŞK 57. C. planisiliqua Fisch. \& C.A.Mey. "telkariotu", (12), 16.04.2008, ŞK 200, (Ir.-Tur.). Descurainia sophia (L.) Webb ex Prantl. subsp. sophia "sadırotu", (6), 16.04.2008, ŞK 168. Draba bruniifolia Steven subsp. bruniifolia var. bruniifolia "kaya dolaması", (7), 16.04.2008, ŞK 121. D. heterocoma Fenzl "tüylü dolama", (21), 30.03.2008, ŞK 111. D. verna L. "çırçırotu", (31), 13.04.2009, ŞK 940, (Ir.-Tur.). Eruca vesicaria (L.) Cav. "roka", (1), 03.05.2009, ŞK 1084. Erysimum crassipes Fisch. \& C.A.Mey. "zarifeotu", (25), 13.06.08 ŞK 470. E. echinelIum Hand.-Mazz. "siğilli zarife", (21), 19.07.2008, ŞK 757, (Ir.-Tur.), (End., EN). E. pycnophyllum J.Gay "yamaç zarifesi", (19), 03.05.2009, ŞK 1029, (Ir.-Tur.). Euclidium syriacum (L.) Aiton "fındık hardalı", (14), 30.03.2008, ŞK 103. Fibigia suffruticus (Vent.) Sweet "mor sikkeotu", (18), 21.06.2009, ŞK 1501. Hesperis bicuspidata (Wild.) Poir. "gecemenekşesi", (13), 18.05.2008, ŞK 320, (Ir.-Tur.), (End., EN). H. syriaca (DC.) Dvorak "arapıtırı", (25), 28.05.2009, ŞK 1298. (E.Medit), (End., VU). H. pendula DC. subsp. pendula "din gildek", (7), 03.05.2009, ŞK 984. Iberis carica Bornm. "kayakbeğendi", (12), 18.05.2008, ŞK 199, (E.Medit.) (End., LR). I. simplex DC. "civanotu", (32), 06.07.2008, ŞK 519. Isatis aucheri Boiss. "pos çiviotu", (13), 18.05.2008. ŞK 315, (Ir. Tur.) (End., LR). I. glauca Aucher ex Boiss. subsp. glauca "soğutot", (1), 19.07.2008, ŞK 772. I. undulata Aucher ex Boiss. "etekli çiviotu", (31), 19.05.2009, ŞK 1134, (Ir. Tur.) (End., EN). Lepidium draba L. "diğnik", (12), 16.04.2008, ŞK 193. L. perfoliatum L., (6), 16.04.2008, ŞK 169. Maresia nana (DC.) Batt. "yanalot" (6), 16.04.2008, ŞK 171. Matthiola longipetala (Vent.) DC. subsp. longipetala "gece- gündüz çiçeği", (9), 28.05.2009, ŞK 1172. M. Iongipetala (Vent.) DC. subsp bicornis (Sibth. \& Sm.) P.W.Ball "boynuzlu şebboy, (10), 28.05.2009, ŞK 1197. M. ovatifolia (Boiss.) Boiss. "takkeli şebboy" (10), 07.05.2013 ŞK 3916, (Ir.-Tur.), (VU). Microthlapsi perfoliatum (L.) F.K.Mey. "giyle", (1), 30.03.2008, ŞK 95. Nasturtium officinale R.Br. "suteresi", (12), 07.05.2013 ŞK 3874. Neslia. paniculata (L.) Desv. subsp. thracica (Velen.) Bornm. "göçmen hardalı", (4), 24.04.2013 ŞK 3678. Neotorularia torulosa (Desf.) Hedge \& J. Léonard "yeni bülbülotu", (9), 03.05.2009, ŞK 1020. N. paniculata (L.) Desv. subsp. thracica (Velen.) Bornm. "göçmen hardalı" (3), 24.04.2013 ŞK 3678. Rapistrum rugosum (L.) All. "kedi turpu", (12), 18.05.2008, ŞK 211. Sisymbrium altissimum L. "ergelenotu" (1), 24.05.2014, ŞK 4868. S. Ioselii L. "bülbülotu", (22), 21.09.2008, ŞK 811. Sterigmostemum incanum M. Bieb. "boz süsün", (10), 28.05.2009, ŞK 1199, (Ir.-Tur.). Strigosella africana (L.) Botsch. "keçe teresi", (20), 18.05.2008, ŞK 87. Tchihatchewia isatidea Boiss. "allıgelin", (2), 18.05.2008, ŞK 236, (Ir.-Tur.), (End., VU). Zuvanda crenulata (DC.) Askerova "davar teresi", (7), 03.05.2009, ŞK 988, (Ir.-Tur.).

\section{CAMPANULACEAE/ÇANÇiçEĞííiLLER (3/10)}

Asyneuma amplexicaule (Willd.) Hand-Mazz. var. angustifolium (Boiss.) Bornm. "hoş değnek", (32), 06.07.2008, ŞK 532, (Ir.-Tur.). A. eximium Rech.f. "düldüldeğneği", (25), 06.06..2009, ŞK 1416, (E. Medit.), (End., LR). A. limonifolium (L.) Janehen subsp. limonifolium "tavşan ekmeği", (18), ŞK 1491. A. limonifolium (L.) Janehen subsp. pestalozzae (Boiss.) Damboldt "tavşankatığı", (32), 06.07.2008, ŞK 527, (End., LR). A. linifolium (Boiss. \& Heldr.) Bornm. subsp. linifolium "ketendeğneği", (23), 06.07.2008, ŞK 568, (E. Medit.), (End., LR). A. lobelioides (Wild.) Hand.-Mazz. "bozkırdeğneği", (19), 13.06.2008, ŞK 446, (Ir.-Tur.). Campanula propinqua Fisch. \& C.A.Mey. "kum çanı", (12), 16.06.2012, ŞK 2269. C. rapunculoides L. "elmacık", (9), 02.11.2008, ŞK 847. C. sclerotricha Boiss. "dere çıngırağı", (13), 06.07.2008, ŞK 641, (Ir.-Tur.). Legousia falcata (Ten.) Fritsch ex Janch "eğri kadınaynası", (10), 24.05.2014, ŞK 4827, (Medit.). L. speculum-veneris (L.) Durande ex Vill. "hoş kadınaynası", (10), 07.05.2013 ŞK 3886, (Medit.). 


\section{CANNABACEAE/KENEVIRGILLER (1/1)}

Celtis planchoniana K.I.Chr. "dahum",

(3),

28.05.2009, ŞK 1272.

\section{CAPRIFOLIACEAE/HANIMELIGILLER (8/21)}

Centranthus longiflorus Steven subsp. Iongiflorus "mahmuz çiçeği", (13), 06.07.2008, ŞK 692, (Ir.-Tur.). Cephalaria syriaca (L.) Schrad. "pelemir", (16), 13.06.2008, ŞK 371. Knautia involucrata Sommier \& Levier "deli eşekkulağı", (31), 21.06.2009, ŞK 1626. Morina persica L. var. persica "merdivençiçeği", (16), 13.06.2008, ŞK 371, (Ir.-Tur). Pterocephalus pinardi Boiss. "yurt cücükotu", (13), 18.05.2008, ŞK 294, (E.Medit.), (End., LR). Scabiosa argentea L. "yazı süpürgesi", (16), 13.06.2008, ŞK 399. S. calocephala Boiss. "çayır uyuzotu", (23), 21.06.2009, ŞK 1550, (Ir.-Tur.). S. persica Boiss. "acem zivanı", (10), 07.05.2013 ŞK 3909, (Ir.-Tur.). S. pseudogrominifolia Hub.-Mor. "çimen uyuzotu", (24), 21.06.2009, ŞK 1592, (Ir.-Tur.), (End., LR). S. rotata M.Bieb. "top uyuzotu", (13), 18.05.2008, ŞK 342, (Ir.-Tur.). Valeriana dioscoridis Sm. "çoban zurnası", (18), 03.05.2009, ŞK 1001, (E. Medit.). V. sisymbriifolia Vahl. "ipar kediotu", (17), 12.05.2007, ŞK 25. Valerianella carinata Loisel "sandal kuzugevreği", (25), 28.05.2009, ŞK 1292. V. coronata (L.) DC. "taçlı kuzugevreği", (10), 24.05.2014, ŞK 4823. V. cymbicarpa C.A.Mey. "azer kuzugevreği", (2), 12.05.2007, ŞK 56, (Ir.-Tur). V. dufresnia Bunge ex Boiss. "acı kuzugevreği", (10), 24.04.2013 ŞK 3708. V. kotschyi Boiss. "tarla kuzugevreği", (7), 28.05.2009, ŞK 1167. V. locusta (L.) Laterr. "nazlı kuzugevreği", (7), 03.05.2009, ŞK 985, (Euro.-Sib.). V. oxyrhyncha Fisch. \& C.A.Mey. "dik kuzugevreği", (10), 24.04.2013 ŞK 3655, (Ir.Tur.). V. pumila (L.) DC. "bağ kuzugevreği", (18), 23.04.2009, ŞK 914. V. vesicaria (L.) Moench "kuzu gevreği", (13), 18.05.2008, ŞK 351.

\section{CARYOPHYLLACEAE/KARANFILGILLER $(14 / 51)$}

Acanthophyllum oppositiflorum Aytaç "has takacak", (18), 20.07.1996, Aytaç 7476, (Ir.-Tur), (End., EN). Arenaria macrosepala Boiss. "toptüyotu", (18), 19.05.2009, ŞK 1117, (End., LR). A. sivasica Kit Tan \& Sorger "sivas kumotu", (24), 09.09.1977, Sorger 77-107-2, (Ir.-Tur.), (End., EN). Bufonia virgata Boiss. "tel hatunotu", (29), 1906, Post 104.
Cerastium chlorifolium Fisch. \& C.A.Mey. "parlak boynuzotu", (31), 19.05.2009, ŞK 1127. C. dichotomum L. subsp. dichotomum "çatal boynuzo$\mathrm{tu}^{\prime \prime},(13), 18.05 .2008$ ŞK 307. c. dichotomum L. subsp. inflatum (Link) Cullen "patlak boynuzotu", (25), 19.05.2009, ŞK 1159. C. dubium (Bastard) O.Schwarz "mızrak boynuzotu", (13), 18.05.2008, ŞK 325. C. gracile Dufour "küçük boynuzotu", (7), 03.05.2009, ŞK 982. C. perfoliatum L. "ekin boynuzotu", (7), 16.04.2008, ŞK 179. Dianthus crinitus Sm. var. crinitus "uzun çanak", (25), 06.06.2009, ŞK 1402. D. micranthus Boiss. \& Heldr. "kır karanfili", (21), 19.07.2008, ŞK 758. D. orientalis Adams "yar karanfili", (10), 28.05.2009, ŞK 1249. D. strictus Banks \& Sol. var. gracilior (Boiss.) Reeve "dimisok", (32), 06.06.2009, ŞK. 1470. D. zederbaueri Vierh. "er karanfil", (24), 21.06.2009, ŞK 1599. D. zonatus Fenzl var. aristatus (Boiss.) Reeve "kaya karanfili", (31), 06.07.2008, ŞK 634, (Ir.-Tur.). Eremogone ledeboriana (Fenzl) Ikonn. "iğne kumotu", (23), 28.05.2009, ŞK 1349, (End., LR). Gypsophila leucochlaena Hub.-Mor. "darende çöveni" (9), Hub.-Mor. 12649, (Ir.-Tur.), (End., EN). G. perfoliata L. var. perfoliata "helvacı çöveni", (3), 28.05.2009, ŞK 1188. G. pilosa Hudson "tarla çöveni", (16), 13.06.2008, ŞK 396. G. pinifolia Boiss. \& Hausskn. "hıskıdım", (10), 24.05.1014, ŞK 4828, (Ir.-Tur.), (End., VU). G. ruscifolia Boiss. "acem çöveni", (9), 28.05.2009, ŞK 1188, (Ir.-Tur.). G. viscosa Murr. "sadırlı çöven", (1), 13.06.2008, ŞK 513, (Ir.-Tur.). Herniaria glabra L. "atyaran", (32), 06.06.2009, ŞK 1469. H. incana Lam. "kabayaran", (10), 24.05.2014, ŞK 5058. Holosteum tenerrimum Boiss. "kaşıkııran", (11), 12.05.2007, ŞK 95. H. umbellatum L. var. umbellatum "şeytan küpesi", (1), 30.03.2008, ŞK 116. H. umbellatum L. var. glutinosum (M.Bieb.) Gay "şeytan küpesi", (7), 16.04.2008, ŞK 55. Minuartia corymbulosa (Boiss. \& Balansa) McNeill var. gypsophiloides McNeill "kırk tıstıs", (19), 13.06.2008, ŞK 440, (Ir.Tur.), (End., EN B2ab(v)). M. glandulosa (Boiss. \& A.Het) Bornm. "sadırlı tıstıs", (10), 24.04.2013 ŞK 3726, (Ir.-Tur.), (End., LR). M. juniperina (L.) Maire \& Petitm. "hanım şiltesi", (19), 13.06.2008, ŞK 444. M. meyeri (Boiss.) Bornm. "koza tıstısı", (25), 06.06.2009, ŞK 1423, (Ir.-Tur.). M. rimarum (Boiss. \& Balansa) Mattf. var. multiflora McNeill "yer tıstısı", (31), 23.04.2009, ŞK 933, (Ir.-Tur.), (End., VU). Paronychia argyroloba Stapf. "taş kepekotu", (23), 06.07.2008, ŞK 571, (End., LR). 
P. cataonica Chaudhri "gürün etyaranı", (21), 19.07.2008, ŞK 769, (End., VU B2ab(ii)). P. euphratica (Chaudhri) Chaudhri "fırat etyaranı", (4), 21.06.2009, ŞK 1529, (Ir.- Tur.), (End., LR). $\boldsymbol{P}$. kurdica Boiss. subsp. haussknechtii Chaudhri "kepekotu" (10), 24.05.2014, ŞK 5034, (End., LR). P. kurdica Boiss. subsp. kurdica var. kurdica "boz kepekotu, (21), 19.07.2008, ŞK 768. Saponaria prostrata Willd. subsp. anatolica Hedge. "ana sabunotu", (23), 06.07.2008, ŞK 617, (Ir.-Tur.), (End., LR). S. prostrata Willd. subsp. prostrata "ebemterliği", (9), 18.05.2008, ŞK 277, (Ir.-Tur.), (End., LR). S. tridentata Boiss. "üşmen", (9), 06.06.2009, ŞK 1368, (Ir.-Tur.). Silene argentea Ledeb "boz nakıl", (25), 21. 06. 2009, ŞK 1644, (Ir.-Tur.). S. armena Boiss. var. armena "çı̆̆ıştak", (21), 19.07.2008, ŞK 770, (End., LR). S. chlorifolia Sm. "puşkullu", (27), Hub.-Mor. 14917, (Ir.-Tur.). S. dichotoma Ehrh. subsp. dichotoma "çatal nakıl", (10), 24.04.2013 ŞK 3696. S. Iongipetala Vent. "ballı süpürge", (13), 18.05.2008, ŞK 308. S. macrodonta Boiss. "deve nakılı", (10), 28.05.2009, ŞK 1242. S. montbretiana Boiss. "Fay nakılı", (30), 19.05.2009, ŞK 1123, (Ir.-Tur.). S. muradica Schischk. "Malazgirt nakılı", (13), 18.05.2008, ŞK 334, (Ir.-Tur.), (End., LR). S. odontopetala Fenzl "kunduzotu", (13), 18.05.2008, ŞK 309. S. pungens Boiss. "yastık nakılı", (10), 10.05.2014, ŞK 4716, (Ir. Tur.). S. spergulifolia (Desf.) M.Bieb. "ana nakılı", (31), 21.06.2009, ŞK 1611. S. stenobotrys Boiss. \& Hausskn. "Maraş nakılı", (19), 13.06.2008, ŞK 437, (Ir.-Tur.). S. supina M.Bieb. subsp. pruinosa (Boiss.) Chowdhuri "fırat nakılı", (23), 21.06.2009, ŞK 1566. Stellaria media (L.) Vill. "kuşotu", (26), 23.04.2009. ŞK 921.

\section{CISTACEAE/LADENGILLER (2/8)}

Fumana aciphylla Boiss. "kır güneşotu", (18), D. 21947, (Ir.-Tur.). F. procumbens (Dunal) Gren. \& Godr. "yer güneşotu", (10), 24.05.2014, ŞK 4813. F. trisperma Hub.-Mor. \& Reese "üç güneşotu", (16), 13.06.2008, ŞK 379, (Ir.-Tur.), (End., EN). Helianthemum germanicopolitanum Bornm. "özge güngülü", (32), 06.07.2008, ŞK 543, (End., EN). $\boldsymbol{H}$. ledifolium (L.) Mill. "kuru güngülü", (10), 10.05.2014, ŞK 4699. H. microcarpum Coss. ex Boiss. "çalı güngülü", (27), 06.06.2009. H. nummularium (L.) Mill. subsp. Iycaonicum Coode \& Cullen "güngülü", (29), D. 21858, (End., LR). H. oelandicum (L.) DC. subsp. incanum (Willk.) G.López "tüylü güngülü",
(10), 10.05.2014, SK 4673.

\section{CONVOLVULACEAE/}

TARLASARMAŞIĞIGILLER (3/10)

Convolvulus cantabrica L. "çadırçiçeği", (10), 28.05.2009, ŞK 1208. C. cataonicus Boiss. \& Hausskn. ex Boiss. "kaplumbağaotu", (10), 28.05.2009, ŞK 1198. , (Ir.-Tur.), (End., LR). C. compactus Boiss. "bodur dolaşgan", (13), 18.05.2008, ŞK 349, (Ir.-Tur). C. galaticus Rost. ex Choisy "boz sarmaşık", (1), 13.06.2008, ŞK 512, (Ir.-Tur.), (End., LR). C. holosericeus M.Bieb. subsp. holosericeus "gündüzsefası", (4), 06.06.2009, ŞK 1380. C. lineatus L. "top yayılgan", (13), 13.05.2009, ŞK 1039. C. scammonia L. "bingözotu", (8), 18.05.2008, ŞK 254, (E. Medit.). C. stachydifolius Choisy. "leksiotu", (4), 06.06.2009, ŞK 1373, (Ir.-Tur.). Cuscuta kotschyana Boiss. subsp. caudata Bornm. \& Schwarz "koç bostanbozan", (25), 06.06.2009, ŞK 1412, (Ir.-Tur.). *Ipomoea purpurea ((L.) Roth. "kahkaha çiçeği", (10), 28.05.2009, ŞK 1220.

\section{CORNACEAE/KIZILCIK (1/1)}

Cornus mas L., (8), 18.05.2008, ŞK 248, (Euro.Sib.).

\section{CRASSULACEAE/DAMKORUĞUGILLER (1/1)}

Sedum album L. "çobankavurgası", (25), 06.06.2009, ŞK 1388.

CUCURBITACEAE/KABAKGILLER (1/1)

*Cucurbita pepo L. "sakız kabağı", (16), 13.06.2008, ŞK 406.

\section{ELAEAGNACEAE/iĞDEGILLER (1/1)}

Elaeagnus angustifolia L. "iğde", (19), 13.06.2008, ŞK 420.

\section{EUPHORBIACEAE/SÜTLEĞENGILLER (1/9)}

Euphorbia altissima Boiss. var. glabrescens Boiss. ex M.s.Khan "kabargaç", (13), 06.07.2009, ŞK 683. E. arvalis Boiss. \& Heldr. "tarla sütleğeni", (13), 06.07.2008, ŞK 670, (Ir.-Tur). E. condylocarpa M.Bieb "gijeletri", (31), 13.04.2009, ŞK 967. E. denticulata Lam. "karasütlük", (18), 23.03.2009, ŞK 899. E. eriophora Boiss. "şiremara", (29), 14.07.1906, B.Post. E. esula L. subsp. tommasiniana (Bertol.) Kuzmanov "eşek sütleğeni", (14), 02.11.2008, ŞK 851. E. herniariifolia Willd. var. herniariifolia "boncuk sütleğeni", (31), 13.04.2009, 
ŞK 929. E. macroclada Boiss. "neblul", (32), 06.07.2008, ŞK 538, (Ir.-Tur). E. szowitsii Fisch. \& Mey. var. kharputens Azn. ex M.S.Khan "urus sütleğeni", (13), 06.07.2009, ŞK 678, (Ir.-Tur.).

\section{FABACEAE/BAKLAGILLER (19/77)}

Astragalus aduncus Willd. "çengel geven", (23), 28.05.2009, ŞK 1346, (Ir.-Tur.). A. anthylloides Lam. "torbalı geven", (18), 21.06.2009, ŞK 1483, (Ir.-Tur.). A. asciocalyx Bunge. "külah geveni", (7), 03.05.2009, ŞK 990, (Ir.-Tur.). A. cadmicus Boiss. "baba geveni", (31), 03.05.2009, ŞK 1122, (End., LR). A. cephalotes Banks. \& Sol. subsp. cephalotes "başlı geven", (10), 07.05.2013 ŞK 3894. A. christianus L. subsp. christianus "dallı geven", (23), 28.05.2009, ŞK 1321. A. chtnocephalus Boiss. \& Balansa "piç geven", (12), Hub.-Mor. 9077, (Ir.-Tur.). A. cretaceus Boiss. "gök geven", (10), 07.05.2013 ŞK 3887, (Ir.-Tur.). A. cymbibracteatus Hub.-Mor. \& D.F.Chamb. "yatık geven", (7), 19.07.2009, ŞK 785. (Ir.-Tur.), (End., LR). A. darendensis Podlech \& Ekici "Darende geveni", (9), 10.06.2015, ŞK 6100, (Ir.-Tur.) (End., CR B2ab(v);D). A. drusorum Boiss. "cadı geveni", (12) 06.08.1850, Clement.s.n. $\boldsymbol{A}$. hirsutus Vahl. "tüylü geven", (19), 13.05.2009, ŞK 1027, (End., LR). A. karamasicus Boiss. \& Balansa "korumaz geveni", (25), 28.05.2009, ŞK 1286, (Ir.Tur.), (End., LR). A. lycius Boiss."bozkır mumu", (10), 28.05.2009, ŞK 1203, (End., LR). A. mesogitanus Boiss. "aydın geveni", (19), 13.05.2009, ŞK 1079, (Ir.-Tur.), (End., LR). A. malatyaensis Podlech "malatya geveni", (4), 04.06.1987, M.Nydegger 42617, (Ir.-Tur.), (End., CR D). A. melitenensis Boiss. "akça geven", (25), 06.06.2009, ŞK 1409, (Ir.-Tur.), (End., LR). A. nitens Boiss. \& Heldr. "parlak geven", (18), 28.05.2009, ŞK 1362, (Ir.-Tur.). A. onobrychis L. "korunga geveni", (12), 07.05.2013 ŞK 3879. A. pennatulus Hub.-Mor. \& D.F.Chamb. "cuni", (18), M.Zohary \& Plitmann 1767-15, (Ir.-Tur.), (End., LR). A. pendulus DC. "SIrık geveni", (23), 28.05.2009, ŞK 1334, (Ir.-Tur.). A. pinetorum Boiss. subsp. pinetorum "babadağ geveni", (13), 16.04.2008, ŞK 144 (Ir.-Tur.). A. podperae Širj. "oğlak geveni", (9), 21.06.2009, ŞK 1513, (Ir.-Tur.). A. scabrifolius Boiss. "gövdesiz geven", (9), 28.05.2009, ŞK 1177, (Ir.-Tur.), (End., CR $\mathrm{B} 1 \mathrm{ab}(\mathrm{v}))$. A. stenosemioides Bornm. \& Mathews "zirve geveni", (32), 06.06.2009, ŞK 1463, (Ir.-Tur.), (End., EN). A. stevenianus DC. var. stevenianus "arıkaldıran", (10), 24.04.2013 ŞK 3715. A. strigil- losus Bunge "sert geven", (31), 23.04.2009, ŞK 944, (Ir.-Tur.). A. tigridis Boiss. "Dicle geveni", (7), 03.05.2009, ŞK 989, (Ir.-Tur.). A. zahlbruckneri Hand.-Mazz. "kubbe geveni", (25), 28.05.2009, ŞK 1285, (Ir.-Tur.), (End., VU). A. zederbaueri Stadlmann "ermenek geveni", (18), 21.06.2009, ŞK 1483, (Ir.-Tur.), (End., LR). A. xylobasis Freyn \& Bornm. "kemaliye geveni", (7), 13.05.2009, ŞK 1092, (End., LR). Ebenus depressa Boiss. \& Balansa "bodur morgeven", (18), 21.06.2009, ŞK 1503, (Ir.-Tur.), (End., LR). E. hausknechti Bornm. ex Hub.-Mor. "Harput morgeveni", (10), 07.05.2013 ŞK 3873, (Ir.-Tur.), (End., LR). E. laguroides Boiss. "morgeven", (19), 13.06.2008, ŞK 443, (Ir.-Tur.), (End., LR). Cicer pinnatifidum Jaub. \& Spach "çakıl nohutu", (12), 07.05.2013 ŞK 3870. Genista albida Willd. "ak borcak", (9), 18.05.2008, ŞK 276. G. aucheri Boiss. "bayır borcağı", (9), 10.06.2015, ŞK 6101, (End., LR). Glycyrrhiza glabra L. var. glandulifera (Waldst. \& Kit) Regel \& Herder "meyan" (26), D. 21889. Hedysarum aucheri Boiss. "altın batalak", (23), 21.06.2009, ŞK 1525, (Ir.-Tur.), (End., VU). H. candissimum Freyn "hoş batalığı", (16), 13.06.2008, ŞK 361, (Ir.-Tur.), (End., LR). H. elagans Boiss. \& Huet "kır batalağı", (32), 06.07.2008, ŞK 541, (Ir.-Tur.). H. pestallozzae Boiss. "horozbaşı", (32), 06.07.2008, ŞK 525, (Ir.Tur.), (End., LR). H. pogonocarpum Boiss. "tay batalığı", (25), 13.06.2008, ŞK 481, (End., LR). H. pycnostachyum Hedge \& Hub.-Mor. "Fırat batalağı", (32), 06.06.2009, ŞK 1475, (Ir.-Tur.), (End., EN). $\boldsymbol{H}$. rotundifolium Boiss. \& Noë "özge batalak", (4), 06.06.2009, ŞK 1384, (Ir.-Tur.), (End., EN). H. varium Willd. subsp syriacum (Boiss.) Townsend "şam batalağı", (13), 18.05.2008, ŞK 300, (Ir.-Tur.). H. varium Willd. subsp. varium "batalak", (23), 28.05.2009, ŞK 1338. Lathyrus blepharicarpus Boiss. "kirpikli imirdiği", (13), 18.05.2008, ŞK 340. Medicago fischeriana (Ser.) Trautv. "mızrak yonca", (8), 13.06.2008, ŞK 511. M. Iupilina L. "bitçik otu", (8), 18.05.2008, ŞK 243. M. monantha (C.A.Mey.) Trautv. "dağ gurniği", (16), 13.06.2008, ŞK 394. (Ir.-Tur.). M. papillosa Boiss. subsp. papillosa "siğilli yonca", (12), 18.05.2008, ŞK 229, (Ir.-Tur.). M. x varia Martyn "yaban yoncası", (1), 16.04.2008, ŞK 186. Melilotus officinalis (L.) Desr. "kokulu yonca", (12), 18.05.2008, ŞK 212. Onobrychis argyrea Boiss. subsp. argyrea "gümüş korunga", (16), 13.06.2008, ŞK 392, (Ir.-Tur.). O. cappadocica 
Boiss. "athelvası", (9), 28.05.2009, ŞK 1175, (Ir.Tur.), (End., LR). O. montana DC. subsp. cadmea (Boiss.) P.W.Ball. "bağ korungası", (1), 24.05.2014, ŞK 4873. O. stenostachya Freyn subsp. krausei (Sirj.) Hedge "başak korunga", (31), 06.07.2008, ŞK 622, (Ir.-Tur.), (End., EN). Ononis hirta Desf. "örsele", (2), 12.05.2007, ŞK 28, (Medit.). O. spinosa L. subsp. leiosperma (Boiss.) Sirj. "demirdelen", (12), 07.05.2013 ŞK 3858. *Phaseolus coccineus L. "bombay fasülyesi", (15), 02.11.2008, ŞK 834. *Pisum sativum L. subsp. elatius (M.Bieb.) Aschers \& Graebn var. pumilio Meikle "boylu bezeIye", (22), 06.07.2008, ŞK 602. *Robinia pseudocacia L. CV. umbraculifera "yalanciakasya", (10), 28.05.2009, ŞK 1175. Securigera varia (L.) Lassen "körigen", (13), 18.05.2008, ŞK 289. Sophora alopecuroides L. var. alopecuroides acımeyan", (10), 10.05.2014, ŞK 4722. Trifolium hybridum L. var. hybridum "melez üçgül", (12), 07.05.2013, ŞK 3880. T. michelianum Savi var. balansae (Boiss.) Azn. "uzundiş", (10), 28.05.2009, ŞK 1217. T. pratense L. var. pratense "çayır üçgülü", (8), 18.05.2008, ŞK 244, 265. T. stellatum L. var. stellatum "yıldız yonca", (10), 24.04.2013 ŞK 3695. Trigonella foenum-graceum L. "çemenotu", (8), 18.05.2008, ŞK 242. T. kotschyi Fenzl "akboyotu", (9), 28.05.2009, ŞK 1162. T. mesopotamica Hub.-Mor. "Dicle boyotu”, (8), 18.05.2008, ŞK 260, (Ir.-Tur). T. spruneriana Boiss. "koç boyotu", (10), 24.04.2013 ŞK 3657. T. velutina Boiss. “ipek boyotu", (7), 19.07.2008, ŞK 714, (Ir.-Tur.) Vicia hirsuta (L.) Gray "bozfiğ", (10), 24.05.2014, ŞK 5045. V. peregrina L. "kavli", (13), 18.05.2008, ŞK 288. V. sativa L. subsp. nigra L.) Ehrh. "eşek gürülü", (16), 13.06.2008, ŞK 368. V. villosa Roth subsp. eriocarpa (Hausskn.) P.W.Ball "boğala", (16), 13.06.2008, ŞK 370.

\section{FAGACEAE/KAYINGILLER (1/1)}

Quercus petraea (Matt.) Liebl. subsp. pinnatiloba (K.Koch) Menitsky "koca pelit", (24), 21.06.2009, ŞK 1573.

\section{GERANIACEAE/TURNAGAGASIGILLER (3/10)} Erodium absinthoides Willd. subsp. armenum (Trautv.) P.H.Davis "kaya iğneliği", (25), 13.04.2009, ŞK 955, (Ir.-Tur.). E. aytacii Yıld. \& Doğru-Koca "bey iğneliği" (16) 13.06.2008, ŞK 375, (Ir.-Tur.), (End., EN B2ab(v)). E. cicutarium (L.) L'Hér. subsp. cicutarium “iğnelik”, (7),
30.03.2008, ŞK 120. E. gaillardotii Boiss. "bozkır iğneliği", (14), 13.05.2009, ŞK 1101, (Ir.-Tur. VU). E. micropetalum Boiss. \& Hausskn. "kokar iğnelik", (10), 24.04.2013, ŞK 3672, (E.Medit.), (End., LR). Geranium dissectum L. "dilimli ıtır", (17), 12.05.2007, ŞK 34. G. purpureum Vill. "ebedön", (9), 02.11.2008, ŞK 844. G. rotundiflorum L. "helilok", (18), 03.05.2009, ŞK 1006. G. tuberosum L. "çakmuz", (1), 18.05.2008, ŞK 326. Pelargonium endlicherianum Fenzl "solucanotu", (31), 19.05.2009, ŞK 1148, (Ir.-Tur.).

\section{HYPERICACEAE/KANTARONGILLER (1/9)}

Hypericum adenotrichum Spach "kızılcıkotu", (21), 13.06.2008, ŞK 494, (End., LR). H. capitatum_Choisy var. Iuteum N.Robson "bantof", (23), 21.06.2009, ŞK 1556, (Ir.-Tur.). H. Iydium Boiss. "cayesanciyan", (16), 13.06.2008, ŞK 426. H. perforatum L. subsp. veronense (Schrank) H.Linb. "sarı kantaron", (7), 19.07.2008, ŞK 713. H. pseudolaeve N.Robson "keşiş kantaronu", (4), 21.06.2009, ŞK 1532, (End., LR). H. retisum Aucher "aran", (18), 28.05.2009, ŞK 1352, (Ir.-Tur.). H. scabrum L. "karahasançayı", (18), 13.06.2008, ŞK 463, (Ir.Tur.). H. thymbrifolium Boiss. \& Noë "bozkır kantaronu", (16), 13.06.2008, ŞK 413, (Ir.-Tur.), (End., LR). H. thymopsis Boiss. "darende kantaronu", (2), 29.11.2008, ŞK 854, (Ir.-Tur.), (End., LR).

\section{JUGLANDACEAE/CEVIZGILLER (1/1)}

Juglans regia L. "ceviz", (13), 18.05.2008, ŞK 305.

\section{LAMIACEAE/BALLIBABAGILLER (18/56)}

Ajuga chamaepitys (L.) Schreb. subsp. chia (Schreber) Arcang. "acıgıcı", (18), 28.05.2009, ŞK 1366. A. salicifolia (L.) Schreb. "sivrimayasıl", (16), 33.06.2008, ŞK 416, (Ir.-Tur.). Clinopodium graveolens (M.Bieb.) Kuntze subsp. graveolens "filiskin", (10), 24.05.2014, ŞK 4833. Cyclotrichium niveum (Boiss.) Manden \& Scheng. "külotu", (16), Sorger 77-105-20, (Ir.-Tur.), (End., VU). Eremostachys moluccelloides Bunge "benli sultan", (18), D. 21868, (Ir.-Tur.). Lallemantia iberica (M.Bieb.) Fisch \& C.A.Mey. "ajdarbaşı", (10), 24.04.2013 ŞK 3683, (Ir.-Tur.). L. peltata (L.) Fisch. \& C.A.Mey. "kalkanbaşı", (19), 13.05.2008, ŞK 1081, (Ir.Tur.). Lamium amplexicaule L. var. aleppicum (Boiss. \& Hausskn. ex Boiss.) Bornm. "baltutan", (21), 30.03.2008, ŞK 112. L. amplexicaule L. var. amplexicaule Boiss. \& Hausskn. "baltutan", (1), 
30.03.2008, ŞK 117, (Euro-Sib.). L. garganicum L. subsp. striacum (Sm.) var. striacum Hayek "tel balıcak", (24), 03.05.2009, ŞK 995. L. orientale Fisch. \& Mey., (10), 13.05.2009, ŞK 1071, (Ir.-Tur.), (End., LR). Marrubium globosum Montbret \& Aucher ex Benth. subsp. globosum "bozcaboğum", (25), 06.06.2009, ŞK 1429, (Ir.-Tur.), (End., LR). M. parviflorum Fisch. \& C.A.Mey. subsp. oligodon (Boiss.) Seybold. "küllü bozotu", (17), 12.05.2008, ŞK 60. M. trachyticum Boiss., "itsineği", (24), Sorger, 77-106-17, (Ir.-Tur.), (End., LR). Mentha Iongifolia (L.) L. subsp. typhoides "dere nanesi" (18), McNeill 427. Micromeria cremnophila Boiss. \& Heldr. subsp. anatolica PH Davis "taş boğumcuğu", (12), D. 21852, (Ir.-Tur.), (End., LR). M. myrtifolia Boiss. \& Hohen "boğumlu çay", (10), 24.05.2014, ŞK 4817. Phlomis armeniaca Willd. "boz şavlak", (32), 06.07.2008, ŞK 536, (Ir.-Tur.), (End., LR). P. linearis Boiss. \& Balansa "yaylaotu", (9), 21.06.2009, ŞK 1521, (Ir.-Tur.), (End., LR). $\boldsymbol{P}$. oppositiflora Boiss. \& Hausskn. "has çalba", (13), 06.07.2008, ŞK 657, (Ir.-Tur.), (End., LR). P. physocalyx Hub.-Mor. "küfe çalbası", (12), Hub.-Mor. 8903, (Ir.-Tur.), (End., VU). P. pungens Willd var. pungens "silvanok", (23), 06.07.2008, ŞK 581. Prunella laciniata (L.) "bodur fesleğen", (30), 06.07.2008, ŞK 627. P. vulgaris L. "gelinciklemeotu", (23), 06.07.2008, ŞK 613, (Euro.-Sib.). Salvia abscontidiflora (Montbret \& Aucher ex Benth.) Greuter \& Burdet "kara şalba", (12), 18.05.2008, ŞK 210, (Ir.-Tur.), (End., LR). S. aethiopis L. "habeş adaçayı", (13), 18.05.2008, ŞK 316. S. ballsiana Rech. f.) Hedge "gerger şalbası", (3), 28.05.2009, ŞK 1192, (Ir.-Tur.), (End., DD). S. cassia Samuelss ex Rech.f. "kel şalba", (12), 03.05.2013 ŞK 3860, (Medit.). S. dichroantha Stapf. "kutnu", (13), 06.07.2008, ŞK 651, (Ir.-Tur.), (End., LR). S. euphratica Montbret \& Aucher var. euphratica "fırat şalbası", (13), 18.05.2008, ŞK 286. S. euphratica Montbret \& Aucher var. leiocalycina (Rech. f.) Hedge "fırat şalbası", (25), 13.06.2008, ŞK 478, (Ir.-Tur.), (End., LR). S. frigida Boiss. "sağır şalba", (16), 13.06.2008, ŞK 422, (Ir.-Tur.). S. hypargeia Fisch. C.A.Mey. "siyahot" (16), 13.06.2008, ŞK 428, (Ir.-Tur.), (End., LR). S. multicaulis Vahl. "kürt reyhanı", (10), 13.05.2009, ŞK 1062. S. palaestina Benth. "sürmeli şalba", (10), 24.04.2013 ŞK 3660, (Ir.-Tur.). S. staminea Montbret \& Aucher ex Benth. "erkek şalba", (21), 19.07.2008, ŞK 756. S. suffruticosa Montbret \& Aucher ex Benth. "kalın şalba", (18), 21.06.2009, ŞK 1492, (Ir.-Tur.). S. verticillata L. subsp. amasiaca (Freyn \& Bornm.) Bornm. "hart şalbası", (22), 06.07.2008, ŞK 616, (Ir.-Tur.). Scutellaria orientalis L. subsp. cretacea (Boiss. \& Hausskn) J.R.Edm. "kulaklı kaside", (16), 13.06.2008, ŞK 360, (Ir.-Tur.), (EN). S. orientalis L. subsp. pectinata (Montbret \& Aucher ex Benth.) J.R.Edm. "taraklı kaside", (16), 13.06.2008, ŞK 427, (Ir.-Tur.), (End., LR). S. orientalis L. subsp. pinnatifida J.R.Edm. "kırbaç sırımı", (16), 28.05.2009, ŞK 1351. Sideritis libanotica Labill. subsp. kurdica (Bornm.) Hub.-Mor. "inceçay", (23), 06.07.2008, ŞK 576, (Ir.-Tur.). Stachys annua L. subsp. annua var. annua "haciosmmanotu", (3), 28.05.2009, ŞK 1274. S. annua L. subsp. annua var. Iycaonica R.Bhattacharjee "dağ çayçesi", (21), 19.07.2008, ŞK 749. S. arvensis (L.) L. "tarla karabaşı", (14), 19.05.2009, ŞK 1103. S. cretica L. subsp. anatolica Rech.f. "yağlıkara", (23), 06.07.2008, ŞK 619, (Ir.-Tur.), (End., LR). S. iberica M.Bieb. subsp. stenostachya (Boiss.) Rech. f. "benli delçay", (2), ŞK 1492, (Ir.-Tur.). S. lavandulifolia Vahl. "tüylü çay", (13), 18.05.2008, ŞK 317, (Ir.-Tur.). S. Iongispicata Boiss. \& Kotschy "ak deliçay", (3), 28.05.2009, ŞK 1274, (Ir.-Tur.). S. ramosissima Montbret \& Aucher ex Benth. "harput deliçayı", (18), Hub.Mor., (Ir.-Tur.), (End., LR). Teucrium chamaedrys L. subsp. sinuatum (Celak) Rech.f. "sanciotu", (26), D. 21955, (Ir.-Tur.). T. chamaedrys L. subsp. syspirense (K.Koch) Rech.f. "kısamahmut", (13), 06.07.2008, ŞK 691. T. multicaule Montbret \& Aucher ex Benth. "haptutan", (16), 13.06.2008, ŞK 412, (Ir.-Tur.). T. orientale L. var. glabrescens Haussckn. ex Bornm. "kirveotu", (8), 18.05.2008, ŞK 272. T. orientale L. var. puberulens Ekim "kirveotu", (13), 18.05.2008, ŞK 298, (Ir.-Tur.). T. polium L. subsp. polium "acıyavşan", (13), 18.05.2008, ŞK 290. Thymus cappadocicus Boiss. "taş kekiği", (25), 06.06.2009, ŞK 1457, (Ir.-Tur.), (End., CR). T. pallasicus Hayek \& Velen "boz kekik", (21), 13.06.2008, ŞK 493 (Ir.-Tur.), (End., CR). T. pectinatus Fisch. \& C.A.Mey. var. pectinatus "sivas kekiği", (32), 06.07.2008, ŞK 540, (Ir.-Tur.), (End., LR). T. sipyleus Boiss. "sipil kekiği", (16), 13.06.2008, ŞK 419. Ziziphora clinopodioides Lam. "dağ reyhanı", (17), 12.05.2009, ŞK 295. Z. persica Bunge "kara reyhan", (9), 18.05. 2008, ŞK 295. Z. tenuior L. "fare otu", (23), 06.07.2008, ŞK 580, (Ir.-Tur.) 
LINACEA/KETENGILLER (1/4)

Linum bienne Mill. "deli keten", (12), 18.05.2008, ŞK 198. L. mucronatum Bertol subsp. armenum (Bordz.) P.H.Davis "Sarıkamış keteni", (32), 06.07.2008, ŞK 545, (Ir.-Tur.). L. nodiflorum L. "yaban keten", (23), 06.07.2008, ŞK 588, (Medit.). L. obtusatum (Boiss.) Stapf "akdağ keteni", (12), 03.05.2013 ŞK 3865, (Ir.-Tur.).

\section{LYTHRACEAE/AKLAROTUGILLER (1/2)}

Lythrum salicaria L. "hevhulma", (22), 06.07.2008, ŞK 604, (Euro.-Sib.). L. virgatum L. "çamur aklarotu", (22), 06.07.2008, ŞK 608, (Euro.-Sib.).

\section{MALVACEAE/EBEGÜMECIGILLER (3/6)}

Alcea biennis Winterl. "fatmaanagülü, (9), 16.04.2008, ŞK 176. A. calvertii (Boiss.) Boiss. "hıraçiçeği", (23), 06.07.2008, ŞK 615, (Ir.-Tur.), (End., LR). A. hohenackeri (Boiss. \& Huet) Boiss. "hevur", (9), 16.04.2008, ŞK 174. A. striata (DC.) Alef. subsp. rufescens (Boiss.) Cullen "yivli hatmi", (21), 21.09.2008, ŞK 803. Hibiscus trionum L. "kekrede", (7), 10.08.2008, ŞK 784. Malvella sherardiana (L.) Jaub. \& Spach "hubazi", (3), 28.05.2009, ŞK 1275.

\section{MORACEAE/DUTGILLER (2/3)}

Ficus carica L. subsp. carica "incir", (13), 18.05.2008, ŞK 333, (Ir.-Tur.). *Morus alba L. "akdut", (22), 06.07.2008, ŞK 596. *M. nigra L. "karadut", (12), 21.06.2009, ŞK 1445.

\section{NITRARIACEAE/ÜZERLIKGILLER (1/1)}

Peganum harmala L. "üzerlik", (6), 19.07.2008, ŞK 732.

\section{OLEACEAE/ZEYTINGILLER (1/1)}

Fraxinus angustifolia Vahl. subsp. angustifolia "sivri dişbudak", (8), 18.05.2008, ŞK 249.

\section{ONAGRACEAE/YAKIOTUGILLER (1/2)}

Epilobium hirsitum L. "hasanhüseyin çiçeği", (22), 06.07.2008, ŞK 601. E. montanum L. "dağyakısı", (22), 06.07.2008, ŞK 607, (Euro.-Sib.).

\section{OROBANCHACEAE/CANAVAROTUGILLER}

$(5 / 9)$

Bungea trifida (Vahl) C.A.Mey. "üç kernekotu", (12), 07.05.2013 ŞK 3878, (Ir.-Tur.). Orobanche minor Sm. "göveotu", (18), 19.05.2009, ŞK 1114. 0. oxyloba Beck "kazıkotu", (24), Sorger 76-21-28. 0. schultzii Mutel "kırk veremotu", (25), 28.05.2009, ŞK 1296. Pedicularis comosa L. var. sibthorpii (Boiss.) Boiss. "hotozlu bitotu", (7), ŞK 1934. Phelipanche aegyptiaca (Pers.) Pomel "dinlendiren", (4), 06.06.2009, ŞK 1373. P. bungeana (Beck) Soják "kernek canavarotu", (12), 03.05.2013, ŞK 3835. P. nana (Noë) Soják "veremotu", (12), 07.05.2013 ŞK 3880. Phelypaea coccinea (M.Bieb.) Poir. "kardaşkanı", (9), 06.06.2009, ŞK 1394, (Ir.-Tur.).

\section{PAPAVERACEAE/HAŞHAŞGiLLER (5/15)}

Fumaria asepala Boiss. "ak şahtere", (13), 13.05.2009 ŞK 1044, (Ir.-Tur.). F. officinalis L. subsp. cilicica "yer sofrası", (13), 06.07.2008, ŞK 652. Glaucium acutidentatum Hausskn. \& Bornm. "tavukgötü", (16), 13.06.2008, ŞK 431, (Ir.-Tur.), (End., LR). G. flavum Crantz "gündürmelâlesi", (2), 18.05.2008, ŞK 236. G. grandiflorum Boiss \& A.Huet subsp. refractum (Nábelek) Mory var. refractum "develâlesi", (18), D. 21945, (Ir.-Tur.). G. leiocarpum Boiss. "gâvur haşhaşı", (2), 06.12.2008, ŞK 869. Hypecoum dimitiatum Delile "boynuzlu kimyon", (1), 16.04.2008, ŞK 183. H. pendulum L. L. "tarla düğmeciği", (9), 03.05.2009, ŞK 1018. Papaver argemone L. subsp. argemone "kum haşhaşı", (2), ŞK 1091. P. macrostomum Boiss. \& A. Huet "minimitçe", (13), 18.05.2008, ŞK 339, (Ir.Tur.). $\boldsymbol{P}$. persicum Lindl, subsp. persicum "acem gelinciği" (13), 06.07.2008, ŞK 646. P. persicum Lindl, subsp. fulvum Kit Tan \& Sorger "kaşıklık", (24), Sorger 71-50-33, (End., VU). P. rhoeas L. "gelincik", (16), 13.06.2008, ŞK 354. P. syriacum Boiss. \& C.I.Blanche "kellinar", (16), 13.06.2008, ŞK 356. P. triniifolium Boiss. "titrekızım", (32), 06.07.2008, ŞK 523, (Ir.-Tur.), (End., LR). Roemeria hybrida (L.) DC. subsp. hybrida "pıtpıtotu", (9), 03.05.2008, ŞK 1014.

\section{PHYLLANTHACEAE/DUVARNOHUTUGILLER} (1/1)

Andrachne telephioides L. "duvarnohutu" (12), 07.05.2013, ŞK 3871.

PLANTAGINACEAE/SiNiROTUGILLER (5/20)

Anarrhinum orientale Benth. "süpürgrotu" (12), 16.06.2012, ŞK 2226. Globularia orientalis L. "küre çiçeği", (23), 06.07.2008, ŞK 593, (Ir.-Tur.). G. trichosantha Fisch. \& C.A.Mey. subsp. tricho- 
santha "köşe yayılımı", (22), 16.04.2008, ŞK 154, (Ir.-Tur.). Linaria antilibanotica Rech.f. "arap nevruzotu", (10), 19.05.2009, ŞK 1210, (Ir.-Tur.). L. chalapensis (L.) Mill. subsp. chalapensis "arap nevruzotu", (19), 13.05.2009, ŞK 1078. L. corifolia Desf. "tarla nevruzotu", (25), 19.05.2009, ŞK 1152, (Ir.-Tur.), (End., LR). L. iconia Boiss. \& Heldr. "konya nevruzotu", (1), 06.06.2009, ŞK 1430, (Ir.-Tur.), (End., LR). L. kurdica Boiss. \& Hohen. subsp. kurdica "sarı nevruzotu", (21), 19.07.2008, ŞK 752, (Ir.Tur.). L. pelisseriana (L.) Mill. "mor nevruzotu", (25), 06.06.2009, ŞK 1420, (Medit). L. simplex DC. "yalın nevruzotu" (10), 07.05.2013 ŞK 3914, (Medit). Plantago lanceolata L. "damarlıca", (12), 18.05.2008, ŞK 213. P. major L. subsp. intermedia (Glib.) Lange "yedidamarotu", (13), 06.07.2008, ŞK 672. Veronica anagalis-aquatica L. "sugedemesi", (8), 19.05.2008, ŞK 745. V. cinerea Boiss. \& Balansa "gümüş maviş", (17), 19.05.2009, ŞK 1112, (E. Medit.), (End., LR). V. hederifolia L. "bahar mavisi", (13), 18.05.2008, ŞK 189. V. jacquinii Bornm. "çalı mavişi", (25), 06.06.2009, ŞK 1433, (Euro.-Sib.). V. polifolia Benth. "dallı maviş", (10), 13.05.2009, ŞK 1065, (Ir.-Tur.), (VU). V. polita Fr. "mavişot", (9), 16.04.2008, ŞK 177. V. thymoides P.H.Davis subsp. pseudocinera M.A.Fisch. "bozkır mavişi", (4), 06.06.2009, ŞK 1379, (Ir.-Tur.), (End., LR). V. triloba Opiz "üç maviş", (13), 03.05.2009, ŞK 1041.

\section{PLATANACEAE/ÇINARGILLER (1/1)}

Platanus orientalis L. "çınar" (12), 28.05.2009, observed ŞK.

\section{PLUMBAGINACEAE/KARDIKENIGILLER (2/5)}

Acantholimon acerosum (Willd) Boiss. var. parvifolium Bokhari "pişik keveni", (25), 13.06.2008, ŞK 480, (Ir.-Tur.) (End., VU). A. damassanum Mobayen "gevrek kardikeni", (18), 03.07.2001, Doğan \& Akaydın 6729, (Ir.-Tur.). A. strigillosum Bokhari "narin kirpiotu", (10), 24.04.2013 ŞK 3669, (Ir.Tur.), (End., VU B2ab(i)). A. venustum Boiss. var. venustum "kınalı kirpiotu", (18), D. 21870. Plumbago europaea L. "karakına", (13), 06.07.2008, ŞK 662, (Euro.-Sib).

\section{POLYGALACEAE/SÜTOTUGILLER (1/3)}

Polygala anatolica Boiss. \& Heldr. "yılan yoncası", (27), 06.06.2009, ŞK 1442. P. pruinosa Boiss. subsp. pruinosa "puslu sütotu", (25), 06.06.2009,
ŞK 1394. P. supina Schreb. subsp. supina "gihaye sipirge", (25), 06.06.2009, ŞK 1305.

POLYGONACEAE/MADIMAKGILLER (3/6)

Atrophaxis billardieri Jaub.\& Spach var. billardieri "teke buğdayı", (23), 28.05.2009, ŞK 1329. A. spinosa L. "devekıran", (29), 1906, Post 7, (Ir.Tur.). Polygonum arenastrum Boreau "bezmeceotu", (17), McNeill 646. P. cognatum Meissn. "madımak", 10.08.2008, ŞK 777. Rumex angustifolius Campd. subsp. macranthus (Boiss.) Rech.f. "kertlice", (9), 28.05.2009, ŞK 1171, (Ir.-Tur.). R. scutatus L. "ekşimen", (13), 06.07.2008, ŞK 653.

\section{PORTULACEAE/SEMIZOTUGILLER (1/1)}

Portulaca oleracea L. "semizotu", (17), 12.05.2007, ŞK 29.

\section{PRIMULACEAE/ÇUHAÇiçEĞiGiLLER (2/3)}

Anagallis arvensis $L$. subsp. arvensis "farekulağı", (13), 06.07.2009, ŞK 667. Androsace maxima L. "tavukkursağı", (13), 16.04.2008, ŞK 145. A. villosa L. "catili", (21), 13.06.2008, ŞK 500, (Euro.-Sib.).

\section{RANUNCULACEAE/DÜĞÜNÇiçEĞiGiLLER}

(7/17)

Adonis aestivalis L. subsp. aestivalis "kandamlası", (13), 18.05.2008, ŞK 335. A. aestivalis L. subsp. parviflora (Fisch ex DC.) N.Busch. "kuşlâlesi", (20), 12.05.2007, ŞK 83. Anemone blanda Schott \& Kotshyi "dağlâlesi", (18), 13.04.2009, ŞK 897. Ceratocephalus falcata (L.) Pers "yelotu", (17), 12.05.2007, ŞK 18. Consolida glandulosa (Boiss. \& Huet) Bornm. "yağlı mahmuz", (16), 13.06.2008, ŞK 362, (Ir.-Tur.), (End., LR). C. oliveriana (DC.) Schrödinger "kıllı mahmuz", (29), 14.07.1906, Post. C. orientalis (J.Gay) Schrödinger "morçiçek", (12), 18.05.2008, ŞK 203. C. scleroclada (Boiss.) Schrödinger var. rigida (Frey. \& Sind.) P.H.Davis "sert mahmuz", (13), 06.07.2008, ŞK 658, (Ir.-Tur.). Delphinium dasystachyum Boiss. \& Balansa "kirli hezaren", (13), 06.07.2008, ŞK 661, (Ir.-Tur.), (End., LR). Nigella arvensis L. var. glauca Boiss. "tarla çörekotu", (25), 13.06.2008, ŞK 473. N. latisecta P.H.Davis "ekin çörekotu", (4), 06.06.2009, ŞK 1382, (Ir.-Tur.). N. oxypetala Boiss. "Fırat çörekotu", (9), 21.06.2009, ŞK 1509, (Ir-Tur.). RanuncuIus argyreus Boiss. "çitemik", (13), 18.05.2008, ŞK 299. R. arvensis L. "mustafaçiçeği", (17), 12.05.2007, ŞK 44. R. constantinopolitanus (DC.) 
d'Urv. "kâğıthane çiçeği", (8), 18.05.2008, ŞK 75. R. cuneatus Boiss. "körükotu", (8), 18.05.2008, ŞK 205. R. kotschyi Boiss. "girit lâlesi", (8), 18.05.2008, ŞK 264. R. trichophyllus Chaix ex Vill. "suluçanak", (20), 12.05.2007, ŞK 95.

\section{RESEDACEAE/GERDANLIKGILLER (1/2)}

Reseda lutea L. var. Iutea "muhabbet çiçeği", (17), 12.05.2007, ŞK 40. R. tomentosa Boiss. var. tomentosa "havlı gerdanlık", (25), 28.05.2009, ŞK 1297, (Ir-Tur.), (End., VU B2ab(v);D1).

\section{RHAMNACEAE/CEHRIGILLER (1/1)}

Rhamnus lycioides L. subsp. oleoides (L.) Jahandiez \& Maire "top cehri", (16), 13.06.2008, ŞK 432.

\section{ROSACEAE/GÜLGILLER (14/30)}

Agrimonia eupatoria L. subsp. asiatica (Juz.) Skalicky "fıtıkotu", (31), 06.07.2008, ŞK 623. Amygdalus communis L. "badem", (13), 16.04.2008, ŞK 163. A. Iycioides Spach. var. Iycioides "behiv", (31), 06.07.2008, ŞK 621. A. orientalis Mill. "payam", (11), 30.03.2008, ŞK 96. *Armeniaca vulgaris Lam. "kayısı", (11), 13.04.2009, ŞK 905. *Cerasus avium (L.) Moench. "kiraz", (7), 16.04.2008, ŞK 173. C. incana (Pal.) Spach var. incana "dağ kirazı", (13), 06.07.2008, ŞK 686, (Ir.-Tur.). C. mahaleb Mill. var. mahaleb "mahlep", (13), 06.07.2008, ŞK 659. C. microcarpa Boiss. subsp. microcarpa "yaban kirazı", (13), 16.04.2008, ŞK 142. C. vulgaris Mill. "vişne", (16), 16.04.2008, ŞK 157. Cotoneaster integerrimus L. "garagat", (16), 13.06.2008, ŞK 423. Crataegus azarolus L. var. azarolus "müzmüldek", (19), 21.09.2008, ŞK 802. c. microphylla K.Koch. subsp. microphylla "kocakarı armudu", (10), 18.05.2008, ŞK 246. (Euro.-Sib.). C. monogyna Jacq. subsp. monogyna "yemişen", (23), 06.07.2008, ŞK 597. C. $x$ sinaica Boiss. "çöl alıcı", (13), 06.07.2008, ŞK 640, (Ir.-Tur.). * ${ }^{*} y-$ donia oblonga Mill. "ayva", (69, 19.07.2008, ŞK 736. Malus sylvestris (L.) Mill. subsp. sylvestris "yaban elması", (16), 13.06.2008, ŞK 373. Potentilla argentea L. "gümüș parmakotu", (31), 21.06.2009, ŞK 1614. $\boldsymbol{P}$. inclinata Vill. "eğri parmakotu", (27), 06.06.2009, ŞK 1444. P. recta "su parmakotu", (16), 13.06.2008, ŞK 382. P. reptans L. "reşatınotu", (10), 28.05.2009, ŞK 1235. P. speciosa Willd. "kaya parmakotu", (1), 22.11.2008, ŞK 852. *Prunus divaricata Ledeb. subsp. divaricata "yunus eriği", (6), 19.07.2008, ŞK 734. Pyrus com- munis L. subsp. communis "bey armudu", (22), 16.04.2008, ŞK 158. Rosa canina L. "kuşburnu", (29), 21.09.2008, ŞK 814. R. hemishaerica J.Herrm. "kadıngöbeği", (23), 28.05.2009, ŞK 1322, (Ir.-Tur.). R. orientalis A.Dupont ex DC. "asker gülü", (18), 21.06.2009, ŞK 1478, (Ir.-Tur.). R. villosa L. "sakız gülü", (9), 02.11.2008, ŞK 845. Rubus sanctus Schreb. "böğürtlen", (7), 10.08.2008, ŞK 782. Sanguisorba minor L. subsp. minor "çayırdüğmesi", (12), 18.05.2008, ŞK 214.

\section{RUBIACEAE/KÖKBAYAGILLER (4/13)}

Asperula glomerata (M.Bieb.) Griseb. subsp. eriantha (Hausskn \& Bornm.) Ehrend. "dağ yumyumotu", (9), 28.05.2009, ŞK 1182. A. glomerata (M.Bieb.) Griseb. subsp. codensata (Ehrend.) Ehrend. var. codensata "sık yumyumotu", (25), 28.05.2009, ŞK 1300. A. stricta Boiss. subsp. latibracteata (Boiss.) Ehrend. "berit belumotu", (21), 19.07.2008, ŞK 780, (Ir.-Tur.), (End., LR). Cruciata articulata (L.) Ehrend. "kulaklı sarılıkotu", (13), 16.04.2008, ŞK 146. C. taurica (Pall. ex Willd.) Ehrend. "kırım güzeli", (13), 16.04.2008, ŞK 143. Galium cilicium Boiss. "yayla yoğurtotu", (3), 28.05.2009, ŞK 1190, (E. Medit.), (End., LR). G. davisii Ehrend. "göksün iplikçiği", (12), 18.05.2008, ŞK 230, (E. Medit.), (End., LR). G. humifusum M.Bieb. "çimen iplikçiği", (13), 06.07.2008, ŞK 643. G. incanum $\mathrm{Sm}$. subsp. elatius (Boiss.) Ehrend. "gür iplikçik", (32), 06.06.2009, ŞK 1472, (Ir.-Tur.). G. rivale (Sibth. \& Sm.) Griseb. "boyluca", (13), 06.07.2008, ŞK 654, (Euro.-Sib.). G. spurium L. var. spurium "arsız iplikçik", (12), 18.05.2008, ŞK 209. G. verum L. subsp. glabrescens Ehrend. "sar। yoğurtotu", (32), 06.07.2008, ŞK 546. G. verum L. subsp. verum "boyalık", (31), 19.05.2009, ŞK 1143. Rubia rotundifolia Banks \& Sol. "bostanboyası", (6), 19.07.2008, ŞK 735, (E.Medit.). R. tinctorum L. "kökboyası", (7), 10.08.2008, ŞK 781, (Ir.-Tur).

\section{RUTACEAE/TURUNÇGILLER" (1/3)}

Haplophyllum cappadocicum Spach "peri sedosu", (2), 28.05.2009, ŞK 1181, (Ir.-Tur). (End., LR). H. myrtifolium Boiss. "murt sedosu", (31), 19.05.2009, ŞK 1137, (Ir.-Tur.), (End., EN). Ruta thesioides Fisch. ex DC "kırsedefotu" (25), 28.05.2009, ŞK 1310.

\section{SALICACEAE/SÖĞÜTGILLER (2/3)}

Populus alba L. var. alba "akkavak", (8), 
18.05.2008, ŞK 247. Salix alba L. subsp. alba "aksöğüt", (20), 12.05.2007, ŞK 74. *S. babylonica L. var. babylonica "salkım söğüt", (22), 16.04.2008, ŞK 191.

\section{SANTALACEAE/GÜVELEKGILLER (2/3)}

Thesium bergeri Zucc. "koru güveleği", (19), 13.06.2008, ŞK 452, (E. Medit.). T. billardieri Boiss. "meşe güveleği", (16), 13.06.2008, ŞK 379. Viscum album L. subsp. album "ökseotu", (1), 23.03.2009, ŞK 889.

\section{SCROPHULARIACEAE/SIRACAOTUGILLER (2/11)}

Scrophularia libanotica Boiss. subsp. libanotica var. libanotica "denekutnu", (13), 06.07.2008, ŞK 639, (E. Medit.). S. rimarum Bornm. "sadırIı sıraca", (13), 06.07.2008, ŞK 650. S. scopolii Hoppe ex Pers. var. scopolii "elköpürten", (24), 21.09.2008, ŞK 819. S. xanthoglossa Boiss. var. decipiens (Boiss. \& Kotschy) Boiss. "serkele", (12), 18.05.2008, ŞK 221. Verbascum caudatum Freyn \& Bornm. "keller sığırkuyruğu", (12), 13.07.1906, G. \& B.Post, (Ir.-Tur.), (End., LR). V. cheiranthifolium Boiss. var. asperulum (Boiss.) Murb. "bozkulak", (14), 02.11.2008, ŞK 850. V. cheiranthifolium Boiss. var. cheiranthifolium "bozkulak", (32), 06.07.2008, ŞK 549. V. lasianthum Boiss. ex Benth. "yünlü sığırkuyruğu", (21), 21.09.2008, ŞK 804. V. natolicum (Fisch \& Mey) Hub.-Mor. "anasığırkuyruğu", (31), 19.05.2009, ŞK 1145, (Ir.-Tur.). V. speciosum Schrader "zelve", (23), 06.07.2008, ŞK 594. V. urceolatum Hub.-Mor. "testi sığırkuyruğu", (23), 28.05.2009, ŞK 1332, (Ir.-Tur.), (End., LR). V. wiedemannianum Fisch. \& C.A.Mey. "mor sığırkuyruğu", (9), 28.05.2009, ŞK 1163, (Ir.-Tur.), (End., LR).

SIMAROUBACEAE/KOKARAĞAÇGILLER (1/1) Ailanthus altissima (Mill.) Swingle "kokarağaç", (2), 06.07.2008, ŞK 689.

\section{SOLANACEAE/PATLICANGILLER (3/4)}

*Capsicum annuum L. "biber", (17), 21.09.2008, ŞK 794. Hyoscyamus niger L. "banotu", (11), 13.05.2009, ŞK 1094. H. reticulatus $L$. "kumacıkotu", (1), 19.07.2008, ŞK 704, (Ir.-Tur). Solanum dulcamara L. "sofur", (13), 06.07.2008, ŞK 656, (Euro.-Sib.).

\section{TAMARICACEAE/ILGINGILLER (1/1)}

Tamarix gracilis Willd. "ince Ilgın", (10), 28.05.2009, ŞK 1229.

\section{THYMELAEACEAE/SIYIRCIKGILLER (2/2)}

Daphne oleoides Schreb. subsp. kurdica (Bornm.) Bornm. "mundarca", (18), 29.05.2009, ŞK 1361. Thymelaea passerina (L.) Coss. \& Germ. "çekem", (29), G. \& B.Post 24.

\section{ULMACEAE/KARAAĞAÇGíLLER (1/1)}

UImus minor Mill. "ova karaağacı", (8), 18.05.2008, ŞK 253.

\section{URTICACEAE/ISIRGANGILLER (2/2)}

Parietaria judaica L. "duvarfesleğeni", (13), 18.05.2008, ŞK 303. Urtica dioica L. subsp. dioica "Isırgan", (22), 06.07.2008, ŞK 600.

\section{VIOLACEAE/MENEKŞEGILLER (1/2)}

Viola modesta Fenzl. "sahra menekşesi", (13), 13.05.2009, ŞK 1043. V. occulta Lehm. "saklı menekşe", (11), 13.04.2009, ŞK 900.

\section{VITACEAE/ASMAGILLER (1/1)}

*Vitis sylvestris C.C.Gmel. "deli asma", (19), 13.06.2008, ŞK 439.

\section{LILIOPSIDA/SÜMBÜL SINIFI (14/55/114)}

\section{AMARYLLIDACEAE/NERGISGILLER (1/9)}

Allium asclepideum Bornm. "koç soğanı", (9), 28.05.2009, ŞK 1162, (Ir.-Tur.), (VU). A. asperiflorum Miscz. "beyaz soğan", (10), 28.05.2009, ŞK 1204, (Ir.-Tur.), (VU). A. atroviolaceum Boiss. "lifli körmen", (10), 21.06.2009, ŞK 1507. *A. cepa L. "soğan", (7), 19.07.2008, ŞK 715. A. myrianthum Boiss. "pak soğan", (31), 21.06.2009, ŞK 1622, (Ir.-Tur.). A. orientale Boiss. "doğu soğanı", (16), 18.05.2008, ŞK 333, (E. Medit.). A. pustulosum Boiss. \& Hausskn. "bey soğanı", (26), Hub.-Mor. 11667, (Ir.-Tur.). A. schoenoprasum L., (2), 06.06.2009, ŞK 1378. A. scorodoprassum L. "peynir sirmosu", (16), 13.06.2008, ŞK 34, (Medit.).

\section{ARACEAE/YILANYASTIĞIGILLER (2/3)}

Eminium intortum (Banks \& Sol.) Kuntze "çarpık yılanbacağı", (7), 03.05.2009, ŞK 982, (Ir.-Tur.). E. rauwolffii (Blume) Schott var. rauwolffii "yılanba- 
cağı", (18), 03.05.2009, ŞK 1004, (E. Medit.). Lemna trisulca L. "yivli sumercimeği", (24), Sorger 73-35-17.

\section{ASPARAGACEAE/KUŞKONMAZGILLER (5/8)}

Asparagus officinalis $L$. subsp. officinalis "kuşkonmaz", (8), 18.05.2008, ŞK 270. Bellevalia speciosa (Pallas ex Georgi) Woronow, (13), 18.05.2008, ŞK 331. Hyacinthella acutiloba K.Perss. \& Wendelbo "sivri sümbül", (21), 30.03.2008, ŞK 109, (Ir.-Tur.), (End., LR). Muscari comosum (L.) Mill. "morbaş", (25), 19.05.2009, ŞK 1154. M. neglectum Guss. ex Ten. "arapüzümü", (1), 30.03.2008, ŞK 93. Ornithogalum montanum Cirilo "dağ akyıldızı", (9), 03.05.2009, ŞK 1019. O. narbonense L. "akbaldır", (13), 18.05.2008, ŞK 329, (Medit.). O. sphaerocarpum A.Kern. "salkım sakarca", (10), 28.05.2009, ŞK 1230.

\section{COLCHICACEAE/ACIÇiĞDEMGILLER (1/1)} Colchicum triphyllum Kunze "öksüzali", (14), 30.03.2008, ŞK 102, (Medit.).

\section{CYPERICACEAE/HASIROTUGILLER (3/5)}

Carex distans L. subsp. distans "sina ayakotu", (10), 28.05.2009, ŞK 1234. C. divisa Huds. "zevzirçimeni", (10), 28.05.2009, ŞK 1240. C. nigra (L.) Reichard subsp. dacica (Heuff.) Soó "rumen ayakotu", (3), 28.05.2009, ŞK 1259. Cyperus capitatus Vand. "şehvetotu", (3), 28.05.2009, ŞK 1260. Scirpoides holoschoenus (L.) Sojak subsp. holoschoenus "vurla", (10), 28.05.2009, ŞK 1243.

\section{IRIDACEAE/SÜSENGILLER (3/9)}

Crocus biflorus Mill. subsp. tauri (Maw) B.Mathew "berfan", (14), 30.03.2008, ŞK 101, (Ir.-Tur.). C. cancellatus Herb. subsp. damescanus (Herb.) B.Mathew "pivok", (14), 9.11.2008, ŞK 870, (Ir.Tur.). C. pallasii Goldb. subsp. pallasii "güzçimi", (14), 29.11.2008, ŞK 872. Gladiolus atroviolaceus Boiss. "kıraç süseni", (9), 18.05.2008, ŞK 293, (Ir.Tur.). G. italicus Mill. "kılıçotu", (2), 13.05.2009, ŞK 1045. *Iris x germanica L. "göksüsen", (8), 13.05.2009, ŞK 1056. I. persica L. "buzala", (29), 30.03.2008, ŞK 107, (Ir.-Tur.). I. sari Schott ex Baker "ana kurtkulağı", (13), 18.05.2008, ŞK 134, (Ir.Tur.), (End., LR). I. schachtii Markgr "kır süseni", (23), 28.05.2009, ŞK 1343, (End., LR).

\section{IXIOLIRIACEAE/KÖPEKOTUGILLER (1/1)}

Ixiolirion tataricum (Pallas) Herbent var. tataricum "köpekotu", (7), 03.05.2009, ŞK 981.

JUNCACEAE/KOFAGILLER (1/1)

Juncus inflexus L. subsp. inflexus "sazak", (3), 28.05.2009, ŞK 1280.

\section{LILIACEAE/ZAMBAKGILLER (3/7)}

Fritillaria assyriaca Baker subsp. assyriaca "donuk lale", (1), 18.05.2008, ŞK 156. F. pinardii Boiss. "mahçup lâle", (30), 13.04.2009, ŞK 969. Gagea fibrosa (Desf.) Schult. \& Schult.f. "tellisarı", (31), 23.04.09, ŞK 948. G. glacialis K.Koch "buz yıldızı", (31), 23.03.2009, ŞK 889, (Ir.-Tur.). G. granatelli (Parl.) Parl. "yediyıldı", (13), 30.03.2008, ŞK 100, (Medit.). G. taurica Steven "bozkır yıldızı", (11), 13.04.2009, ŞK 907, (Ir.-Tur.). Tulipa armena Boiss. var. armena "dağ lâlesi", (19), 13.05.2009, ŞK 1073, (Ir.-Tur.).

\section{ORCHIDACEAE/SALEPGILLER (1/1)}

Orchis palustris Jacq. subsp. palustris "çayır salebi", (25), 28.05.2009, ŞK 1301.

\section{POACEAE/BUĞDAYGiLLER (28/58)}

Aegilops biuncialis Vis. "ikikılçık", (8), 18.05.2008, ŞK 256. A. cylindrica Host. "kirpikli ot", (10), 28.05.2009, ŞK 1251, (Ir.-Tur.). A. geniculata Roth. "konbaş", (13), 06.07.2008, ŞK 668. A. umbellulata Zhuk. "hanım buğdayı", (16), 13.06.2008, ŞK 358, (Ir.-Tur.). Agrostis gigantea Roth. "koca tavusotu", (24), Sorger 77-101-14, (Euro.-Sib.). Amblyopyrum muticum (Boiss.) Eig., var. muticum "narin buğday", (29), 14.07.1906, B.Post. Arhenatherum elatius (L.) P.Beauv. var. elatius "çayıryulafı", (8), 28.05.2009, ŞK 1176, (Euro.Sib.). Avena barbata Pott ex Link subsp. barbata "narin yulaf", (16), 13.06.2008, ŞK 389, (Medit.). A. eriantha Durieu "koru yulafı", (9), 06.06.2009, ŞK 1201. A. wiesti Steudel "farazotu", (9), 06.06.2009, ŞK 1371. Brachypodium distachyon (L.) P.Beauv “tekkılcan, (2), ŞK 13.05.2009, ŞK 1089. Briza maxima L. "kuşyüreği", (10), 28.05.2009, ŞK 1232. Bromus danthoniae Trin. subsp. danthoniae "ibubukotu", (4), McNeill 431. B. erectus Hudson "dik brom", (32), 06.07.2008, ŞK 531. B. hordeaceus L. subsp. hordeaceus "başakotu", (18), 13.06.2008, ŞK 464. B. intermedius Guss. "damiyeotu", (32), 06.07.2008, ŞK 553. B. japonicus Thunb. subsp. japonicus "iyeotu", (8), 18.05.2008, ŞK 258. B. 
scoparius L. "ibubuk ekini", (3), ŞK 1263. B. sterilis L. "sağır ilcan", (32), 06.07.2008, ŞK 551. B. tectorum L. "kır bromu", (12), 18.05.2008, ŞK 216. B. tomentellus Boiss. subsp. tomentellus "bozkır bromu" (10), 24.04.2013 ŞK 3703, (Ir.-Tur.). Calamagrostis epigejos (L.) Roth. "bekârotu", (10), 28.05.2009, ŞK 1246. C. pseudophragmites (Haller) Koeler "sazçimi", (22), 06.07.2008, ŞK 611, (Euro.-Sib.). Dactylis glomerata subsp. glomerata "domuzayrığı", (16), 13.06.2008, ŞK 383. Daschampsia caespitosa (L.) P.Beauv. "çayırsaçı", (9), 06.06.2009, ŞK 1372. Echinaria capitata (L.) Desf. "dikenbaşotu", (10), 28.05.2009, ŞK 1207. Elymus erosiglumis Melderis "özge buğday", (23), 06.07.2008, ŞK 598, (Ir.-Tur.), (End., LR). Eremopyrum orientale (L.) Jaup \& Stach "acem tarağı", (9), 28.05.2009, ŞK 1184, (Ir.-Tur.). Festuca anatolica Markgr.-Dann. subsp. anatolica "yurt yumağı", (32), 06.06.2009, ŞK 1466, (End., LR). F. arundinacea Schreb. subsp. arundinacea "kamış yumağı", (25), 28.05.2009, ŞK 1290. Hordeum bulbosum L. "boncuk arpa", (10), 24.04.2013 ŞK 3700. H. murinum L. "pisipisiotu", (8), 18.05.2008, ŞK 255. H. spontaneum K.Koch "yabani arpa", (30), 06.07.2008, ŞK 628. H. vulgare L. "arpa", (4), 13.05.2009, ŞK 1090. Koeleria eriostachya Pan i "yayla kırnalı", (3), 28.09.2009 ŞK 1265. Loliolum subulatum (Banks \& Sol.) Eig "köseçim", (12), 03.05.2013 ŞK 3855, (Ir.-Tur.). Melica ciliata L. subsp. ciliata "kirpikli inci", (13), 06.07.2008, ŞK 647. M. penicillaris Boiss. \& Balansa "sülün inceotu", (30), 06.07.2008, ŞK 630, (Ir.-Tur.). M. persica Kunth subsp. persica "acem inciotu", (16), 13.06.2008, ŞK 352. Phleum boissieri Bornm. "yayla itkuyruğu", (29), 14.07.1906, B.Post, (Ir.Tur.). P. exaratum Griseb. "meşe itkuyruğu", (20), 12.05.2007, ŞK 90. P. montanum C.Koch subsp. montanum "dağ itkuyruğ", (33), 06.06.2009, ŞK 1448. P. pratense L. "çayır itkuyruğu", (20), 13.06.2008, ŞK 465, (Euro.-Sib.). Phragmites australis (Cav.) Trin. ex Steud. "kamış", (10), 28.05.2009, ŞK 1244, (Euro.-Sib.). Poa alpina L. susp. fallax F.Herm. "yayla salkımotu", (13), 16.04.2008, ŞK 135. P. bulbosa L. "yumrulu salkIm", (20), 12.05.2007, ŞK 91. P. timoleontis Heldr. ex Boiss. "gür salkımotu", (2), 13.05.2009, ŞK 1050. P. trivialis L. "kaba salkımotu", (24), Sorger, 77-101-16. Setaria glauca (L.) P.Beauv."sıçansaçı",
(9), 10.06.2015, ŞK 6090. *S. italica (L.) P.Beauv. "kirpi darıs", (23), 06.07.2008, ŞK 573. S. viridis (L.) P.Beauv. "yeşil sıçanotu", (16), 22.09.2008, ŞK 793. Stipa ehrenbergiana Trin. \& Rupr. "sorguçotu", (24), Sorger 71-50-29, (Ir.-Tur.). S. holosericea Trin. "dirgen kılaç", (16), 13.06.2008, ŞK 436, (Ir.-Tur.). Taeniatherum caput-medunsa subsp. asper (Simonk.) Melderis "eğri kılçık", (10), 28.05.2009, ŞK 1238, (Ir.-Tur.). *Triticum aestivum L. "ekmeklik buğday, (16), 13.06.2008, ŞK 388. Vulpia ciliata Dumort "kirpikliçim", (26), Stn. \& Hend. 5247. V. persica (Boiss \& Buhse) Krecz. \& Bobrov "acem kirpikliçimi", (10), 28.05.2009, ŞK 1236, (Ir.-Tur.). *Zea mays L. subsp. mays "mısır", (1), 19.07.2008, ŞK 699.

\section{POTAMOGETONACEAE/SUSÜMBÜLÜGILLER}

(2/2)

Groenlandia densa J.Gay "suteleği", (24), Sorger 77-101-27. Stuckenia pectinatus (L.) Börner "sutarağı", (9), McNeill 453.

\section{TYPHACEAE/SAZGILLER (2/3)}

Sparganium erectum L. subsp. erectum "kındıra", (8), 19.07.2008, ŞK 738, (Euro.-Sib.). Typha minima Funck "aksaz", (10), 28.05.2009, ŞK 1241. T. shuttleworthi W.D.J.Koch \& Sond. "puf sazI", (8), 28.05.2008, ŞK 250.

\section{XANTHORRHOEACEAE/ÇiRişGiLLER (2/6)}

Asphodeline brevicaulis (Bertol.) J.Gay ex Baker subsp. brevicaulis "çirişine", (21), 13.06.2008, ŞK 506. A. damascena (Boiss.) Baker. subsp. damascena "çekiçlik", (13), 18.05.2008, ŞK 282, (Ir.Tur.). A. damascena (Boiss.) Baker. subsp. rugosa E. Tuzlaci “elbizotu”, (23), 06.07.2008, ŞK 599, (E. Medit.), (End., LR). A. taurica (Pall.) Endl. "kılçiriş", (33), 06.06.2009, ŞK 1458, (E. Medit.). A. tenuior (Fisch. ex Bieb.) Ledeb. subsp. tenuiflora (K.Koch) Tuzlacı var. tenuiflora "tesbihcik", (4), Tuzlacı, (Ir.-Tur.). Eremurus cappadocicus J.Gay ex Baker "mor çiriş", (18), Hub.-Mor. 16032, (Ir.-Tur.). E. spectabilis M.Bieb. "çiriş", (31), 19.05.2009, ŞK 1143, (Ir.-Tur.).

\section{ACKNOWLEDGEMENTS}

This study was supported by İnönü University Scientific Research Unit (2008/44). 


\section{References}

1. N. Özhatay, A. Byfield, S. Atay, Türkiye'nin 122 Önemli Bitki Alanı, WWF Türkiye Doğal Hayatı Koruma Vakfı, İstanbul, 2005

2. Taşınmaz Kültür ve Tabiat Varlıkları Tescili, T.C. Resmî Gazete, 19813, 13 Mayıs 1988 (1988) 63,

3. P.H. Davis (ed), Flora of Turkey and The East Aegean Islands, vol: 1-9, Edinburgh: Edinburgh University Press, 1965-1985.

4. Y. Akman, İklim ve Biyoiklim, Palme Yayın. Ankara, 1990.

5. P.H. Davis, K. Tan, R.R. Mill (eds), Flora of Turkey and The East Aegean Islands, v: 10, Edinburgh: Edinburgh University Press, 1988.

6. A. Güner, N. Özhatay, T. Ekim, \& K.H.C. Başer (eds), Flora of Turkey and The East Aegean Islands, vol: 11, Edinburgh: Edinburgh University Press, 2000.

7. T.G. Tutin, V.H. Heywood, N.A. Burges, D.H. Valentine, S.M. Walters \& D.A. Webb (eds), Flora Europaea. vol: 1-5. Cambridge: Cambridge University Press, 19641980.

8. Z. Aytaç, A new species of Acanthophyllum (CaryophyIlaceae) from Central Anatolia, Turkey, Nord. J. Bot. 21 (2001) 263-266.

9. Ş. Yıldırımlı, D.A. Koca, A New species from Turkey, Erodium aytacii (Geraniaceae), Ot Sis. Bot. Derg. 11 (2004) 1-6.
10. H.Yıldırım, Taraxacum rupicolum (Asteraceae): Doğu Anadolu'dan yeni bir Karahindiba (Taraxacum F.H.Wigg.) türü, Bağbahçe Bilim Dergisi, 1:3 (2014) 7281.

11. M. Doğan, G. Akaydın, Synopsis of Turkish Acantholimon Boiss. (Plumbaginaceae), Botanical Journal of the Linnean Society, 154 (2007) 397-419.

12. N. Özhatay, S. Kültür, B. Gürdal, Check-List of Additional Taxa to The Supplement Flora of Turkey VI, J. Fac. Pharm. İstanbul, 43 (2013) 33-82.

13. R.K. Brummitt \& C.E. Powell (eds), Authors of Plant Names. Kew. Royal Botanic Gardens, 1992.

14. A. Güner, S. Aslan, T. Ekim, M. Vural, M.T. Babaç (eds), Türkiye Bitkileri Listesi (Damarlı Bitkiler). İstanbul, Turkey: Nezahat Gökyiğit Botanik Bahçesi ve Flora Araştırmaları Derneği Yayını. İstanbul. 2012.

15. T. Ekim, M. Koyuncu, M. Vural, H. Duman, Z. Aytaç, N. Adıgüzel, Türkiye Bitkileri Kırmızı Kitabı (Pteridophyta ve Spermatophyta), Ankara: TTKD ve Van 100. Yıl Üniversitesi Yayını, 2000.

16. IUCN, Guidelines for Using the IUCN Red List Categories and Criteria: Version 8.1. Prepared by the Standards and Petitions Subcommittee of the IUCN Species Survival Commission, IUCN, Gland, Switzerland and Cambridge. UK, (2010). 